\title{
In vitro and in vivo Anti-Tumor Effects of Pan-HER Inhibitor Varlitinib on Cholangiocarcinoma Cell
} Lines

This article was published in the following Dove Press journal: Drug Design, Development and Therapy

\author{
Hasaya Dokduang ${ }^{1,2}$ \\ Wassana Jamnongkarn ${ }^{1,2}$ \\ Bundit Promraksa ${ }^{2,3}$ \\ Manida Suksawat ${ }^{3}$ \\ Sureerat Padthaisong ${ }^{2,3}$ \\ Malinee Thanee ${ }^{1,2}$ \\ Jutarop Phetcharaburanin ${ }^{1-3}$ \\ Nisana Namwat (D) $^{1-3}$ \\ Sakkarn Sangkhamanon $\mathbb{D}^{2,4}$ \\ Attapol Titapun ${ }^{1,2,5}$ \\ Narong Khuntikeo (D) ${ }^{1,2,5}$ \\ Poramate Klanrit (iD) ${ }^{1-3}$ \\ Watcharin Loilome ${ }^{1-3}$ \\ 'Cholangiocarcinoma Screening and Care \\ Program (CASCAP), Faculty of Medicine, \\ Khon Kaen University, Khon Kaen 40002, \\ Thailand; ${ }^{2}$ Cholangiocarcinoma Research \\ Institute, Khon Kaen University, Khon \\ Kaen 40002, Thailand; ${ }^{3}$ Department of \\ Biochemistry, Faculty of Medicine, Khon \\ Kaen University, Khon Kaen 40002, \\ Thailand; ${ }^{4}$ Department of Pathology, \\ Faculty of Medicine, Khon Kaen \\ University, Khon Kaen 40002, Thailand; \\ ${ }^{5}$ Department of Surgery, Faculty of \\ Medicine, Khon Kaen University, Khon \\ Kaen 40002, Thailand
}

Correspondence: Watcharin Loilome Department of Biochemistry, Faculty of Medicine, Khon Kaen University, Khon Kaen 40002, Thailand

Tel/Fax +66 43348386

Email watclo@kku.ac.th
Background: Cholangiocarcinoma (CCA) is a slowly progressing but highly aggressive malignancy. Targeting the HER protein family represents a potential therapeutic strategy for CCA treatment. The pan-HER inhibitor varlitinib is being developed for the treatment of breast cancer, gastric cancer, and biliary tract cancer, which includes CCA. This study aims to evaluate the anti-tumor effect of varlitinib on CCA using both in vitro and in vivo models. Materials and Methods: HER family expression profiles and the cytotoxic activity of varlitinib were determined in CCA cell lines (KKU-214, KKU-213, KKU-156 and KKU-100) and cholangiocyte (MMNK-1). Anti-proliferation and apoptosis induction were examined in KKU-214 and KKU-100 cell lines. A combination of varlitinib with PI3K inhibitor, BKM-120, was explored for efficacy in the KKU-100 cell line. In addition, the anti-tumor activity of varlitinib on CCA and the key metabolites were evaluated in tumor tissues from CCA xenograft model.

Results: Elevated expressions of EGFR and HER2 were observed in KKU-214 and KKU100 cells and varlitinib can suppress CCA cell growth in the micromolar range. Varlitinib inhibits cell proliferation and enhances cell death via the suppression of Akt and Erk1/2 activity in the KKU-214 cell line. While KKU-100 cells showed a poor response to varlitinib, a combination of varlitinib with BKM-120 improved anti-tumor activity. Varlitinib can significantly suppress tumor growth in the CCA xenograft model after oral administration for 15 days without noticeable toxicity, and aspartate can be the key metabolite to correlate with varlitinib response.

Conclusion: Our study indicates that varlitinib is a promising therapeutic agent for CCA treatment via the inhibition of EGFR/HER2. The anti-tumor effect of varlitinib on CCA also showed synergism in combination with PI3K inhibition. Aspartate metabolite level was correlated with varlitinib response. Combination of varlitinib with targeted drug or cytotoxic drug was recommended.

Keywords: cholangiocarcinoma, varlitinib, pan HER inhibitor, HER receptor family

\section{Introduction}

Cholangiocarcinoma (CCA) is an aggressive malignancy arising from the bile duct epithelia. It is a rare cancer globally, but it is the most common cancer in northeast Thailand where it is associated with liver fluke (Opisthorchis viverrini; Ov) infection. ${ }^{1,2}$ $\mathrm{CCA}$ is a slow progression cancer but its prognosis is very poor due to the diagnostic difficulty at the early stage and a number of patients only undergo treatment when the tumor proceeds to advanced and aggressive stage. The dominant treatment approach for CCA is surgical resection, however, only patients who present with early-stage disease 
are considered for surgery, and complete surgical resection is often followed by local recurrence with a low 5-year survival rate. ${ }^{3-5}$ Moreover, chemotherapy and radiotherapy do not improve the patient's survival rate. ${ }^{6}$ Therefore, the identification of a potential therapeutic regimen for CCA is necessary.

The human epidermal growth factor receptor (HER) family is a tyrosine kinase receptor family that consists of four members: HER1 (EGFR), HER2 (c-erbB-2), HER3 (cerbB-3) and HER4 (c-erbB4). These receptor kinases activate numerous downstream growth factor signaling pathways that regulate diverse cellular processes. ${ }^{7,8}$ In particular, aberrant expression and/or activation of the HER receptor family have been reported in many cancer types, including CCA. ${ }^{9-11}$ Our previous study demonstrated the involvement of the HER receptor family in CCA, especially EGFR, which was found to be activated in both CCA cell lines and patients' tissues. ${ }^{12}$ Moreover, a recent study reported the overexpression of EGFR in human CCA tissues associated with poor survival of CCA patients, and that targeting EGFR with nimotuzumab, an anti-EGFR mAb, can inhibit CCA cell metastasis. ${ }^{13}$ In addition, HER2 amplification was recently reported in liver fluke related CCA. ${ }^{14}$ Based on this information, the HER receptor family represents a promising molecular target for the development of anti-cancer therapeutic agents against CCA.

Varlitinib (ASLAN001) is a small molecule pan-HER inhibitor targeting EGFR, HER2 and HER4 which is being developed as a therapeutic agent for several cancers, including gastric cancer, pancreatic cancer, colorectal cancer, breast cancer and biliary tract cancer (BTC, which includes CCA). Varlitinib has received orphan drug designation for biliary tract cancer by the US FDA and by the Korean Ministry of Food and Drug Safety. ${ }^{15-18}$ Our study aims to evaluate the pre-clinical efficacy of varlitinib in CCA. Here we determined growth inhibitory effect as well as safety of varlitinib in CCA. Moreover, tumor metabolism after varlitinib treatment was carried out regarding to identify the metabolic markers and metabolic pathways that associated with varlitinib response in CCA animal model. The information obtained can be used as basic data to potentially support the development of varlitinib for CCA treatment.

\section{Materials and Methods}

\section{Cell Culture}

Human CCA cell lines derived from different histological types of primary CCA tumor including human CCA cell lines KKU-214, KKU-213, KKU-156, KKU-100 and immortalized transformed cholangiocyte, MMNK-1. Cell lines were obtained from the specimen bank from Cholangiocarcinoma Research Institute and the use of cell lines had ethical approval by Cholangiocarcinoma Research Institute, Khon Kaen University. KKU-214, KKU-213 and KKU-156 were cultured in Dulbecco's modified eagle medium (Sigma-Aldrich, MO, USA), while KKU-100 and MMNK-1 were cultured in Ham' F12 medium (Gibco ${ }^{\circledR}$, CA, USA). Both medias contained $10 \%$ fetal bovine serum (Sigma-Aldrich, MO, USA), $100 \mathrm{U} / \mathrm{mL}$ penicillin, and 100 $\mu \mathrm{g} / \mathrm{mL}$ streptomycin (Sigma-Aldrich, MO, USA). All cell lines were incubated at $37^{\circ} \mathrm{C}$ in a humidified atmosphere containing $5 \% \mathrm{CO}_{2}$.

\section{Antibodies and Reagents}

Antibodies for Western blot were as follows: mouse antiEGFR and mouse anti-HER4 (Abcam, Cambridge, UK), rabbit anti-HER3, mouse anti-p85 PI3K, rabbit anti-phospho EGFR (Try1068), rabbit anti-HER2, rabbit anti-cyclinD1, mouse anti-CDK4, rabbit anti-phospho p44/42 MAPK (pErk1/2), rabbit anti-p44/42 MAPK (Erk1/2), rabbit anti-Akt (Cell signaling technology, Danvers, MA), rabbit anti-phospho HER2 and rabbit anti-phospho Akt (Ser473) (Sigma-Aldrich, MO, USA). Recombinant human EGF was purchased from R\&D systems, MN, USA. Varlitinib was supplied by ASLAN Pharmaceuticals, Singapore. BKM-120 was purchased from Active Biochem, NJ, USA. These were dissolved as stock in $100 \%$ dimethyl sulfoxide (DMSO) and stored at $-80^{\circ} \mathrm{C}$ until use.

\section{Western Blot Analysis}

Cells were harvested and lysed in NP-40 lysis buffer (150 $\mathrm{mM} \mathrm{NaCl}, 50 \mathrm{mM}$ Tris $\mathrm{pH} 8.0$ and 1\% NP-40) containing protease inhibitor cocktail (Roche, Penzberg, Germany) on ice. The lysates were centrifuged at $14,000 \mathrm{rpm}$ at $4^{\circ} \mathrm{C}$ for $10 \mathrm{~min}$. Protein lysates $(40 \mu \mathrm{g})$ were solubilized in SDS buffer and boiled for $5 \mathrm{~min}$ and the supernatants collected. Proteins in the supernatants were determined by BCA protein kit assay (Thermo Fisher Scientific, IL, USA). Protein samples were electrophoresed on $10 \%$ polyacrylamide gels and transferred to polyvinylidene fluoride membranes. The membranes were blocked with $5 \%$ skim milk in Tris-buffered saline (TBS) at room temperature for $1 \mathrm{hrs}$ and incubated with primary antibody for EGFR, HER2, HER3, HER4, phospho-EGFR, phospho-HER2, phosphop44/42 MAPK, p44/42 MAPK, phospho-Akt, Akt at $4^{\circ} \mathrm{C}$ overnight. After rinsing with TBS containing $0.1 \%$ polyoxyethylene sorbitan monolaurate (Tween-20 or TBS-T), 
the membranes were incubated with horseradish peroxidase-conjugated secondary antibody (Invitrogen, CA, USA) at room temperature for $1 \mathrm{hrs}$. Human $\beta$-actin was used as a loading control. After rinsing with TBS-T, the membranes were exposed to the ECL Prime Western Blotting Detection System (GE Healthcare, UK). The immunoblot was analyzed by the ImageQuantTM analysis system (GE Healthcare, UK). The densities of the bands were determined using Image $\mathrm{J}$ software version $1.46 \mathrm{r}$ (Wayne Rasband, NIH).

\section{Cell Viability Assay}

Cell lines (KKU-214, KKU-213, KKU-156, KKU-100 and MMNK-1) $2 \times 10^{3}$ per $100 \mu \mathrm{L}$ were seeded into 96-well plates and incubated overnight at $37^{\circ} \mathrm{C}$ and $5 \% \mathrm{CO}_{2}$. Cells were treated with varlitinib at designated concentrations for $24 \mathrm{hrs}, 48 \mathrm{hrs}$ and $72 \mathrm{hrs}$. Varlitinib was dissolved in 100\% DMSO and added to culture media at different concentrations: the final concentration of DMSO was $0.5 \%$. The number of viable cells was determined using sulforhodamine B (SRB, Sigma-Aldrich, MO, USA). Cells were fixed with $10 \%$ cold trichloroacetic acid for $1 \mathrm{hr}$ at $4{ }^{\circ} \mathrm{C}$ and stained with $0.4 \% \mathrm{w} / \mathrm{v} \mathrm{SRB}$ in $1 \% \mathrm{v} / \mathrm{v}$ acetic acid for 30 mins. Excess dye was washed with $1 \%$ acetic acid and stained cells were solubilized with $200 \mu \mathrm{L}$ of $10 \mathrm{mM}$ unbuffered Tris-base. The absorbance was measured with a microplate reader (Sunrise, TECAN Trading, Switzerland) at $540 \mathrm{~nm}$. The percentage of growth inhibition $(\% \mathrm{GI})$ was calculated using the formula, $\% \mathrm{GI}=(1-(\mathrm{Nt}-$ $\mathrm{Nc}) \times 100$ ) where $\mathrm{Nt}$ and $\mathrm{Nc}$ are the absorbance of the treated and control groups, respectively.

\section{Clonogenic Survival Assay}

Five hundred cells of CCA cell lines KKU-214 and KKU100 were seeded in 6 well plates with medium containing $2.5,5$, or $10 \mu \mathrm{M}$ concentrations of varlitinib or control medium (0.5\% DMSO). After $72 \mathrm{hrs,} \mathrm{the} \mathrm{medium} \mathrm{was}$ removed and replaced with normal medium. Cells were cultured for an additional 14 days then fixed in $4 \%$ paraformaldehyde and stained with crystal violate. Colonies of 50 or more cells were counted.

\section{Caspase 3/7 Activity Assay}

Apoptosis induction in CCA cells was performed using the caspase-GloVR 3/7 assay (Promega) according to the manufacturer's instructions. Briefly, cells $\left(2 \times 10^{3}\right)$ were plated in $100 \mu \mathrm{L}$ of media containing varlitinib at 2.5, 5, or $10 \mu \mathrm{M}$ or control medium and incubated for $72 \mathrm{hrs}$.
Caspase-GloVR 3/7 reagent $(50 \mu \mathrm{L})$ was added and mixed to each well, followed by 30 mins incubation at room temperature. Luminescence was measured on a SpectraMaxL (MDS Analytical Technologies) as per the manufacturer's instructions. Experiments were performed three times with three replicates per experiment.

\section{Flow Cytometry Analysis}

CCA cell lines (KKU-214 and KKU-100) were seeded at $2.5 \times 10^{5}$ cells in a $10 \mathrm{~cm}$ dish and cultured overnight after which the cells were treated with $2.5,5$, or $10 \mu \mathrm{M}$ of varlitinib or control medium for 72 hrs. The cell cycle distribution was then detected by staining DNA with propidium iodide (Invitrogen, Paisley, UK), while apoptosis and necrosis were detected using an Annexin-VFLUOS staining kit (Roche, Penzberg, Germany). The cell cycle distribution and apoptosis were determined by flow cytometry (BD FACSCanto ${ }^{\mathrm{TM}}$ II, BD Biosciences, CA, USA) and analyzed by BDFACSDiva ${ }^{\mathrm{TM}}$ software (BD Biosciences, CA, USA).

\section{Combination of Varlitinib with BKM- 120 in KKU- 100 Cell}

KKU-100 cells $\left(2 \times 10^{3}\right)$ were seeded in 96-well plates overnight then treated with $2.5,5$, or $10 \mu \mathrm{M}$ of varlitinib, varlitinib combined with $2 \mathrm{nM}$ of BKM-120 or control medium for 72 hrs before cell proliferation was determined by SRB assay. The combination index was calculated using the ChouTalalay method with Compusyn software (ComboSyn, Inc., USA). Cell apoptosis was detected by Annexin-VFLUOS staining using flow cytometry analysis. Briefly, $2.5 \times 10^{5}$ KKU-100 cells were seeded in a $10 \mathrm{~cm}$ dish, cultured overnight, and then treated with $2.5,5$, or $10 \mu \mathrm{M}$ of varlitinib, at the designed concentration combined with $2 \mathrm{nM}$ of BKM-120 or control medium for 72 hrs. For molecular analysis, KKU-100 were seeded at $4 \times 10^{5}$ cells in a $10 \mathrm{~cm}$ dish, cultured overnight, and then treated with $2.5,5$, or $10 \mu \mathrm{M}$ of varlitinib at the designed concentration combined with $2 \mathrm{nM}$ of BKM-120 or control medium for $24 \mathrm{hrs}$. After that cells were induced with $100 \mathrm{ng} / \mathrm{mL}$ of EGF for $30 \mathrm{mins}$ before harvesting for protein extraction. The activity of EGFR, HER2 and Akt were determined using Western blot analysis.

\section{Animal Study}

Six-week-old female BALB/cAJcl-nu/nu mice were purchased from Nomura Siam International (Bangkok, Thailand). Animals were housed under specific pathogenfree conditions at the Northeast Laboratory Animal Center 
(NELAC), Khon Kaen University, Thailand. KKU-214 cells in log-phase growth were harvested on the day of use. Cells were suspended in 50:50 serum-free medium/Matrigel (Sigma-Aldrich, MO, USA) and $0.1 \mathrm{~mL}\left(1 \times 10^{6}\right.$ cells $)$ was injected subcutaneously into each animal on both flanks. Ten days after CCA cell injection, the mice were randomized into 4 groups of 6 animals each for oral administration. The control group was orally provided a vehicle (1:9 NMP/Polyethylene glycol 300), while treatment groups were administrated varlitinib at $25 \mathrm{mg} / \mathrm{kg}$ of BW, $50 \mathrm{mg} / \mathrm{kg}$ of BW or $100 \mathrm{mg} / \mathrm{kg}$ of BW, PO, daily for 15 days. Tumors were measured twice each week and volumes calculated by the formula $\left(a \times b^{2}\right) / 2$, where "a" and "b" represent the largest and smallest tumor diameter, respectively. Relative tumor volume was calculated from tumor volume $(\mathrm{V}) /$ tumor volume day $0\left(\mathrm{~V}_{0}\right)$. At the end of the experiment, the mice were sacrificed and tumor tissues, blood and other organs were collected for further investigation.

All procedures were performed in accordance with the guidelines of the Northeast Laboratory Animal Center (NELAC), Khon Kaen University, Thailand, and were approved (AEKKU 28/2559) by the Animal Ethics Committee of Khon Kaen University, Thailand.

\section{Immunohistochemistry}

To determine the anti-proliferative effect of varlitinib using the CCA xenograft model, immunostaining of Ki67 was performed on paraffin-embedded nude mouse tumor tissues. Nude mouse tumor tissue sections were deparaffinized in xylene followed by rehydration in a series of different ethanol concentrations. Then, the antigen was retrieved using Tris-EDTA buffer, $\mathrm{pH} 8.8$, in a pressure cooker, and $0.3 \% \mathrm{H}_{2} \mathrm{O}_{2}$ was used to block endogenous peroxidase activity for $30 \mathrm{mins}$ with shaking. Non-specific binding was blocked by $10 \%$ skim milk in phosphatebuffered saline (PBS) for $30 \mathrm{~min}$. Sections were incubated with rabbit anti-Ki67 antibody (Abcam, Cambridge, UK), dilution 1:200, at $4^{\circ} \mathrm{C}$ overnight in a moisture chamber. Sections were then incubated with peroxidase-conjugated EnVision $^{\mathrm{TM}}$ secondary antibody (Dako, Denmark) followed by washing three times with working PBS for 5 min. Next, the color was developed with $0.1 \%$ diaminobenzidine tetrahydrochloride solution for 5 mins followed by counterstaining with Mayer's hematoxylin. Sections were observed under a light microscope (Nikon H600L, Nikon, Japan). Ki67-positive cells of each tumor section were counted in at least five $\mathrm{x} 400$ power fields.

\section{TUNEL Assay}

To assess the percentage of apoptotic cells in CCA nude mouse tissues, an in situ terminal deoxynucleotide transferase-mediated dUTP nick-end labeling (TUNEL) assay was performed using the DeadEnd ${ }^{\mathrm{TM}}$ Colorimetric Apoptosis Detection System (Promega, WI, USA) according to the manufacturer's instructions. TUNEL-positive cells were quantified in at least five $\mathrm{x} 400$ power fields of randomly selected tissue sections.

\section{Toxicity Assay}

To study the adverse effects of varlitinib in treated mice, they were continuously observed for appearance parameters such as weight loss, diarrhea, anorexia, skin ulcer or death. After sacrifice, the organs, including lung, liver, kidney and spleen, were stained with H\&E, and subjected to histological analysis by a pathologist. The levels of liver enzymes (ALT and AST), as well as creatinine in serum, were determined with an automatic multifunction-biochemical analyzer (Automate Chemistry LX-20 pro, Beckman Coulter).

\section{Metabolite Extraction and NMR Analysis}

In brief, $100 \mathrm{mg}$ of mouse tumor tissue from animal study described above were freshly collected and quenched using liquid nitrogen. Then, lysates were centrifuged by vacuum concentrator. After that, the lyophilized material was dissolved with NMR buffer, transferred into the $5 \mathrm{~mm}$ NMR tube; then, the proton NMR spectra was analyzed using a $400 \mathrm{MHz}$ NMR spectrometer (Bruker, USA). The NMR spectra was aligned and plotted using MATLAB program (Mathworks, USA). The statistical total correlation spectroscopy (STOCSY) analysis method was performed to aid the metabolite assignment in the ${ }^{1} \mathrm{H}$ NMR spectra acquired. Here, the metabolites were identified to match the correlation peak with the literature, Biological Magnetic Resonance Data Bank (BMRB), and the Human Metabolome Database (HMDB). Fold change was calculated as the log transformation of the ratio between the mean metabolite intensities of varlitinib-treated mice tissues relative to the control untreated mice tissue. The Student's $t$ test was performed for statistical analysis among each treatment group versus the control group. A P-value $<0.05$ was considered as statistically significant. To observe the metabolic profiling of tissue, the peak intensity of each of the metabolites was calculated and heatmap analysis based on Pearson's correlation was then 
performed with pathway analysis using Metscape and Cytoscape.

\section{Statistical Analysis}

The results from cell proliferation, Ki67 staining analysis, apoptosis assay and animal experiments are represented as mean $\pm \mathrm{SD}$; statistical significance was determined by oneway ANOVA and two-way ANOVA (GraphPad Prism 5 software). A P-value of $<0.05$ was considered to indicate a statistically significant result.

\section{Results}

\section{HER Receptor Expression Profiles in CCA Cell Lines}

The expression level of the HER protein family was determined using Western blot analysis in four CCA cell lines: KKU-214, KKU-213, KKU-156 and KKU-100. MMNK-1 was also used as the reference cholangiocyte. The results showed that the highest expression levels of EGFR and HER2 were found in KKU-214 cell followed by KKU-100 and KKU-213 while low expression levels were determined in KKU-156 and MMNK-1. The expression of HER3 was most prominently detected in KKU-214 and KKU-213 cells and was not observed in other cell lines, HER4 expression was also detected in the tested cell lines at lower levels, as demonstrated in Figure 1.

\section{Cytotoxic Effect of Varlitinib on CCA Cell Lines}

We then examined whether varlitinib could inhibit CCA cell proliferation. CCA cell lines (KKU-214, KKU-213, KKU-156 and KKU-100) and the reference cholangiocyte, MMNK-1 were treated with a range of concentrations of the inhibitor, and cell proliferation was assessed using SRB assay. The results showed that varlitinib effectively suppressed CCA cell growth at micromolar concentrations in a dose-dependent manner (Figure 2). The $\mathrm{IC}_{50}$ values (mean $\pm \mathrm{SD}$ ) of varlitinib in the four CCA cell lines KKU214, KKU-213, KKU-156, KKU-100, MMNK-1 were $4.83 \pm 0.35 \mu \mathrm{M}, 5.10 \pm 0.44 \mu \mathrm{M}, 4.5 \pm 0.52 \mu \mathrm{M}, 7.68 \pm 0.39$ $\mu \mathrm{M}$ and $9.13 \pm 1.42$, respectively.

We found that the $\mathrm{IC}_{50}$ of varlitinib in KKU-214, KKU213 and KKU-156 cells fell within a similar range, KKU-100 cells showed a poor response with higher $\mathrm{IC}_{50}$ values than other CCA cell lines, while MMNK-1 cell showed higher $\mathrm{IC}_{50}$ over CCA cells. These indicate a non-toxic effect of varlitinib on non-malignant cholangiocyte.

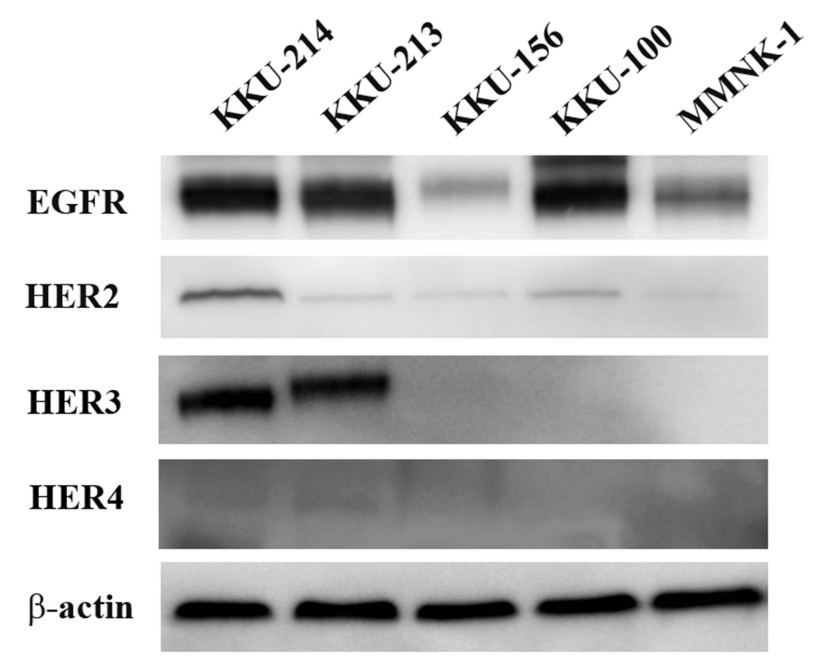

Figure I HER receptor family basal expression in cholangiocarcinoma cell lines and immortalized transform cholangiocyte.

Notes: The expression of EGFR, HER2, HER3 and HER4 was detected in four CCA cell lines (KKU-214, KKU-2I3, KKU-I56 and KKU-I00) and MMNK-I cell use as the reference cholangiocyte by Western blot analysis.

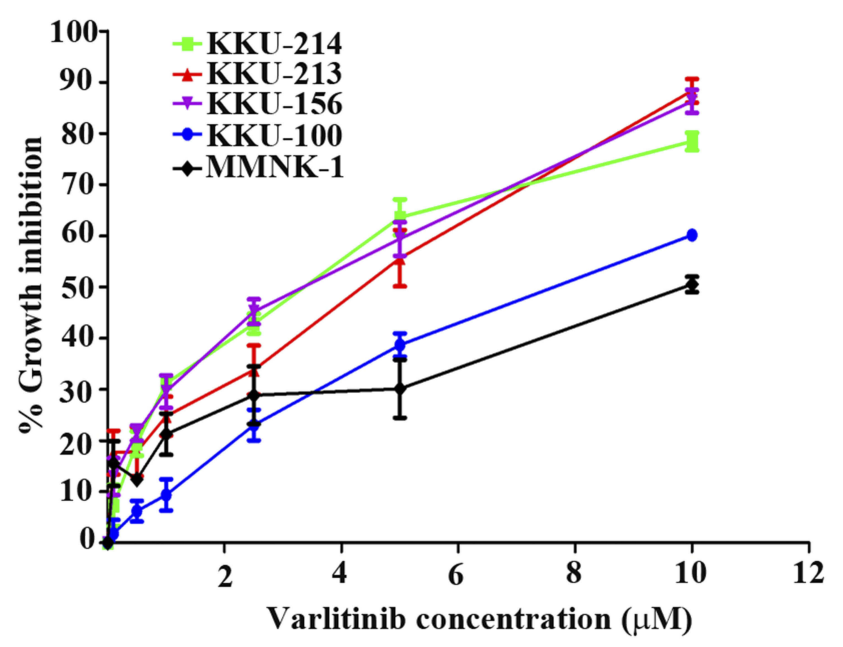

Figure 2 The cytotoxic effect of varlitinib on CCA cells and cholangiocyte. Notes: Four CCA cell lines and nonmalignant cholangiocyte were treated with varlitinib at concentrations ranging from 0.1 to $10 \mu \mathrm{M}$ in $0.5 \%$ DMSO for $72 \mathrm{hrs}$. After incubation, cellular proteins of the viable cells were measured using the sulforhodamine B assay.

According to our findings, the protein expression levels of EGFR, HER2 and HER3 were prominently found in KKU-214 and EGFR, HER2 in KKU-100 cell lines were high; however, the response to varlitinib was different. Therefore, these two cell lines were selected for further study.

\section{Anti-Proliferation Activity of Varlitinib on CCA Cell Lines}

To determine the growth inhibitory effect of varlitinib on CCA cells, the cells were exposed to varlitinib at $2.5 \mu \mathrm{M}$, 
$5 \mu \mathrm{M}$ or $10 \mu \mathrm{M}$ for $72 \mathrm{hrs}$ before a clonogenic survival assay was performed. The results showed that varlitinib significantly inhibited colony formation of KKU-214 cells in a dose-dependent manner, whereas in KKU-100 cells, only $10 \mu \mathrm{M}$ of varlitinib significantly suppressed colony formation (Figure 3A and B). Moreover, flow cytometry analysis demonstrated that varlitinib significantly induced G1 arrest, as well as reducing S phase progression of the CCA cell lines, for KKU-214 in a dose-dependent manner and for KKU-100 at $10 \mu \mathrm{M}$ (Figure $3 \mathrm{C}$ and D). The expression of the G1/S cell cycle regulatory proteins cyclinD1 and CDK4 in KKU-214 after treatment with varlitinib was dramatically decreased in a dose-dependent manner, while this was slightly decreased for CDK4 at 10 $\mu \mathrm{M}$ in KKU-100 (Figure 3E and F).

\section{Apoptotic Induction of Varlitinib on CCA Cells}

To test whether varlitinib inhibited cell growth, at least in part, by inducing apoptosis, cells were treated with 2.5, 5 or $10 \mu \mathrm{M}$ of varlitinib for $72 \mathrm{hrs}$. Untreated cells or varlitinib-treated cells were stained with annexin $\mathrm{V}$ and PI and then analyzed using flow cytometry. The results revealed that varlitinib enhanced KKU-214 cell death in a concentration-dependent manner (both early apoptosis and late apoptosis), and showed significantly induced apoptosis at $10 \mu \mathrm{M}$, while varlitinib-treated KKU-100 cells showed slight apoptosis as shown in Figure 4A and B.

Moreover, we tested whether the mediator of apoptosis, caspase 3/7, was induced. As represented in Figure 4C, there was a slight increase of caspase $3 / 7$ activity in KKU214 treated with $10 \mu \mathrm{M}$ of varlitinib, whereas there was no significant change in caspase $3 / 7$ activity in varlitinib treated KKU-100 cells.

\section{Molecular Mechanisms Underlying Varlitinib Inhibition of CCA Cell Growth}

To explore the molecular mechanisms by which varlitinib inhibits CCA cell growth, cells were pre-treated with various concentrations of varlitinib $(2.5,5$ or $10 \mu \mathrm{M})$ for $24 \mathrm{hrs}$ then induced with $100 \mathrm{ng} / \mathrm{mL}$ of EGF (R\&D system, MN, USA) for 30 mins. The results showed that varlitinib could inhibit EGFR and HER2 activation upon EGF stimulation in KKU-214 cells, while activation of EGFR and HER2 in KKU-100 cells were only inhibited at with $10 \mu \mathrm{M}$ of varlitinib (Figure $5 \mathrm{~A}$ and $\mathrm{B}$ ). We also investigated the downstream signaling pathways of EGFR/
HER2. We focused on the Ras/MAPK and PI3K/Akt pathways that have been reported as two majors signaling cascades implicated in tumor development and triggered by EGFR/HER2 activation. Our results demonstrated that a low concentration of varlitinib $(2.5 \mu \mathrm{M})$ caused an increase in Erk1/2 activation, while $5 \mu \mathrm{M}$ and $10 \mu \mathrm{M}$ inhibited Erk1/2 activity in EGF induced KKU-214 and KKU-100 cells (Figure 5C and D). Varlitinib inhibited Akt phosphorylation at $5 \mu \mathrm{M}$ and $10 \mu \mathrm{M}$, while there was a slight elevation of phospho-Akt at low concentration (2.5 $\mu \mathrm{M})$ in EGF induced KKU-214 cells. On the other hand, phospho-Akt expression in EGF induced KKU-100 cells was not decreased after treatment with varlitinib (Figure 5C and D).

\section{Combination of Varlitinib with BKM-I 20 to Potentiate the Cytotoxic Effect on the KKU-I00 Cell Line}

As demonstrated above, the KKU-100 CCA cells showed high expression of EGFR and HER-2; however, they showed a poor response to varlitinib when compared with KKU-214 cells, which also highly expressed EGFR and HER-2. Moreover, Western blot analysis revealed that the poor response of KKU-100 cells may be caused by an alternative signaling pathway that renders KKU-100 cells resistant to Akt modulation by varlitinib treatment. Therefore, a combination of varlitinib with the PI3K inhibitor BKM-120 was tested for its anti-tumor effect. Our results showed that the combination of varlitinib with BKM-120 exerted a synergistic effect on KKU-100 cell growth inhibition as represented in Figure 6A and Table S1. In addition, flow cytometry analysis demonstrated that BKM-120 significantly potentiated the cytotoxic effect of varlitinib in a dose-dependent manner (Figure 6B and C). As shown in Figure 6D and E, after KKU-100 was exposed to varlitinib combined with BKM120 , there was a decrease in phospho-EGFR and phosphoAkt, where phospho-Akt was further decreased in the combination treatment compared to BKM-120 treatment alone. Conversely, activation of the HER2 receptor was still observed.

\section{Anti-Tumor Activities of Varlitinib Using the CCA Xenograft Model}

To evaluate the in vivo anti-tumor effect of varlitinib on CCA, the CCA inoculated mouse model was used. KKU214 was subcutaneously injected into athymic BALB/c 
A

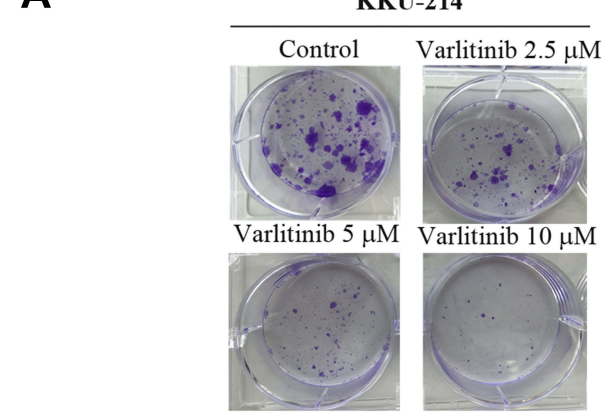

B

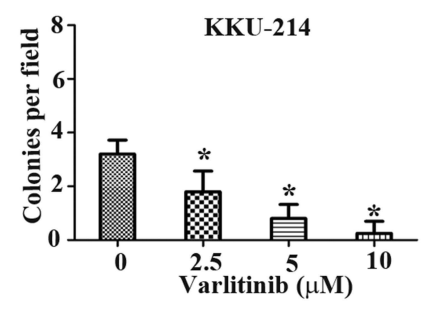

C

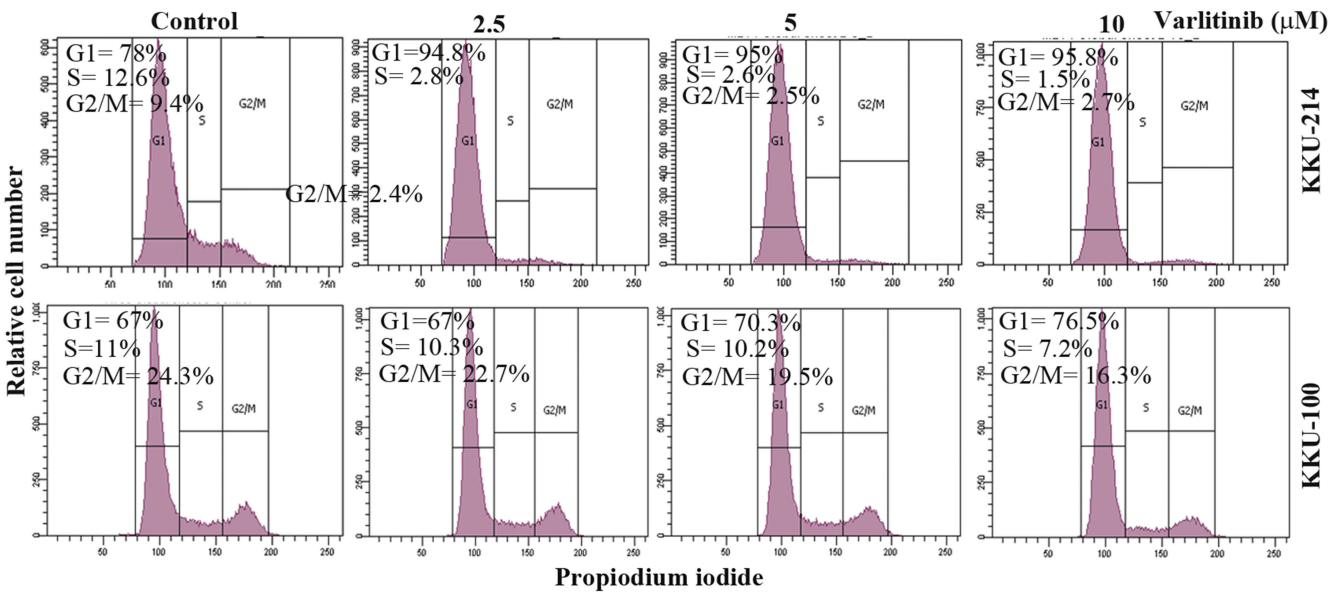

D

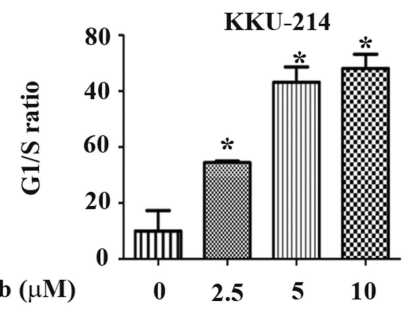

E

Varlitinib $(\mu \mathrm{M})$

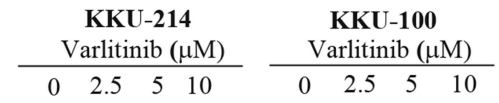

cyclin D1 $\begin{array}{llll}0 & 2.5 & 5 & 10\end{array}$

CDK4

$\beta$-actin

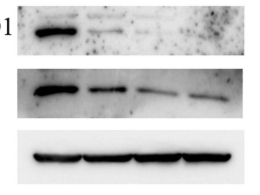

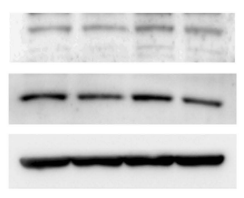
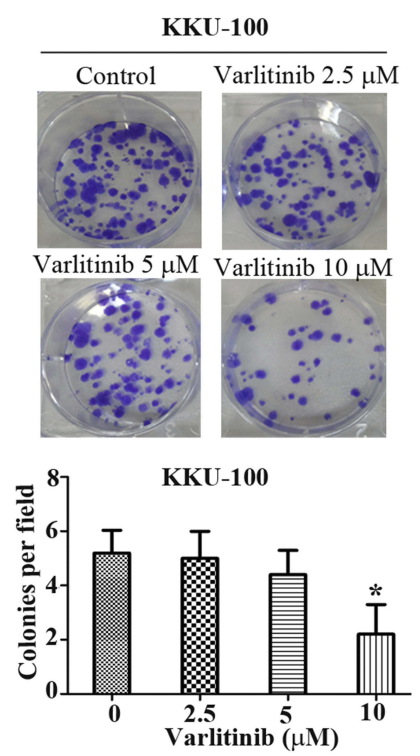
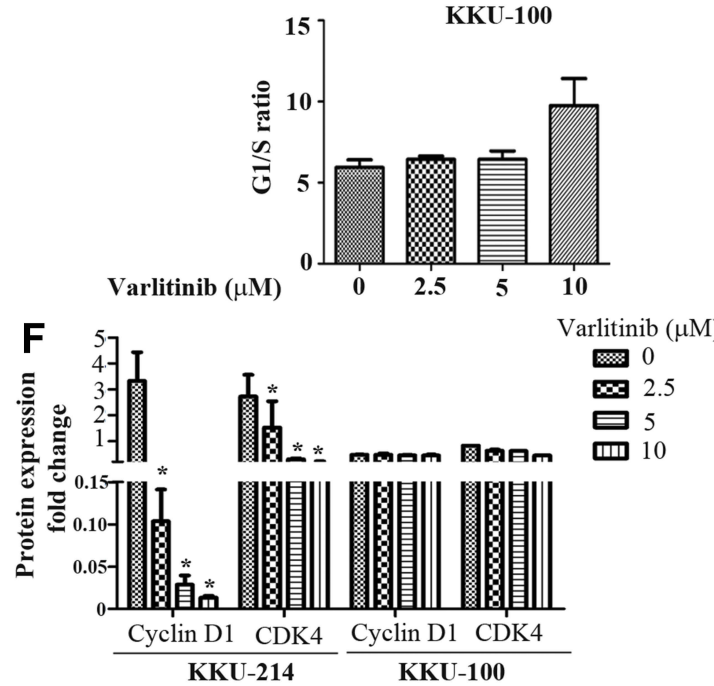

Figure 3 Varlitinib inhibits CCA cell proliferation.

Notes: (A and B) KKU-214 and KKU-100 CCA cells were treated with varlitinib for 72 hrs before the growth inhibitory effect was determined by a clonogenic survival assay: colonies which contained $>50$ cells were counted. (C and D) Varlitinib caused cell cycle arrest in the GI phase. KKU-2I4 and KKU-I00 were treated with varlitinib for 72 hrs and subjected to propidium iodide staining analysis. (E) GI/S phase cell cycle regulatory proteins cyclin DI and CDK4 expression levels were determined by Western blot analysis after KKU-2I 4 and KKU- 100 cells were exposed to varlitinib for 72 hrs. (F) Expression level of cyclin DI and CDK4 after normalized with $\beta$-actin. Data in (B, D and F) are represented as mean \pm SD of three independent experiments. Asterisks indicate statistical significance $(P<0.00 \mathrm{I})$ compared to the control, analyzed by one-way ANOVA. 
A

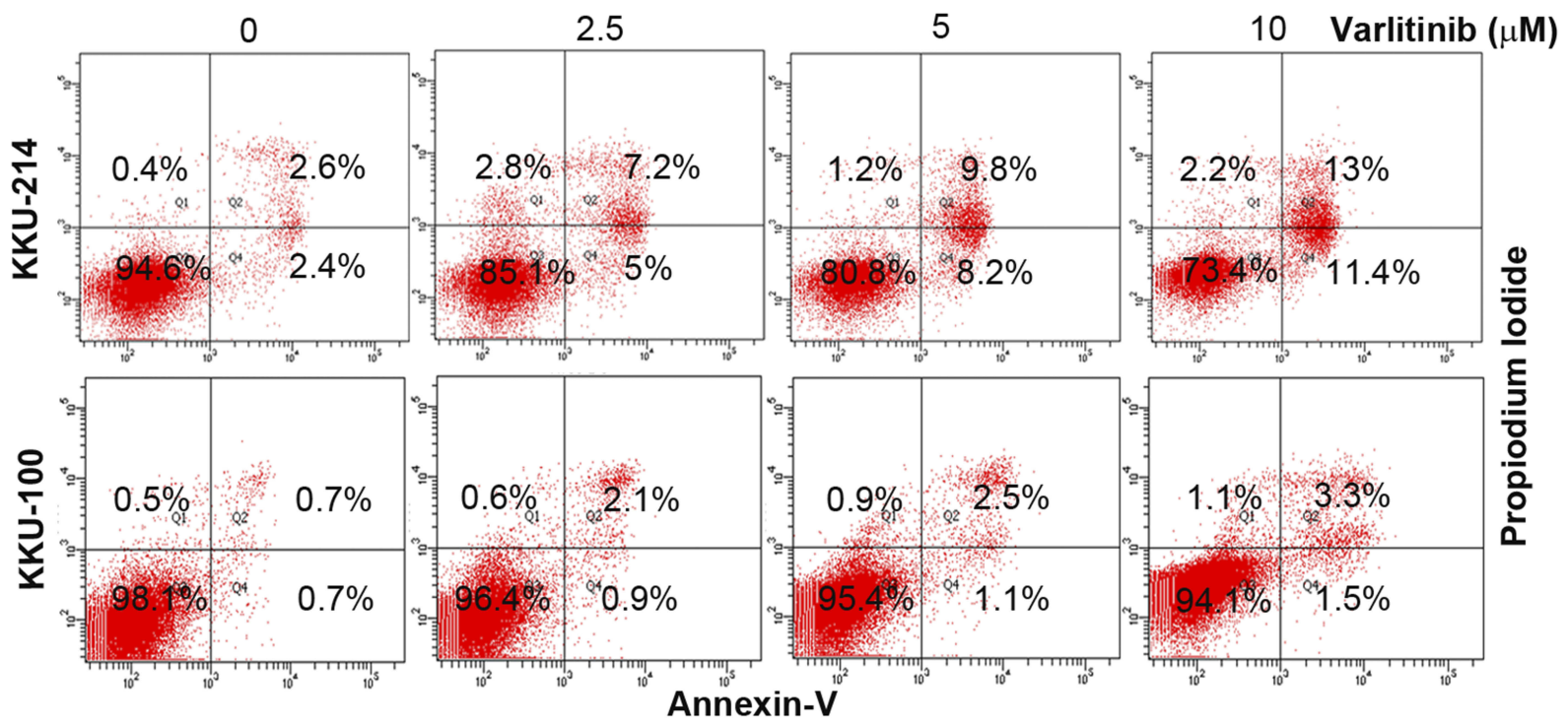

B
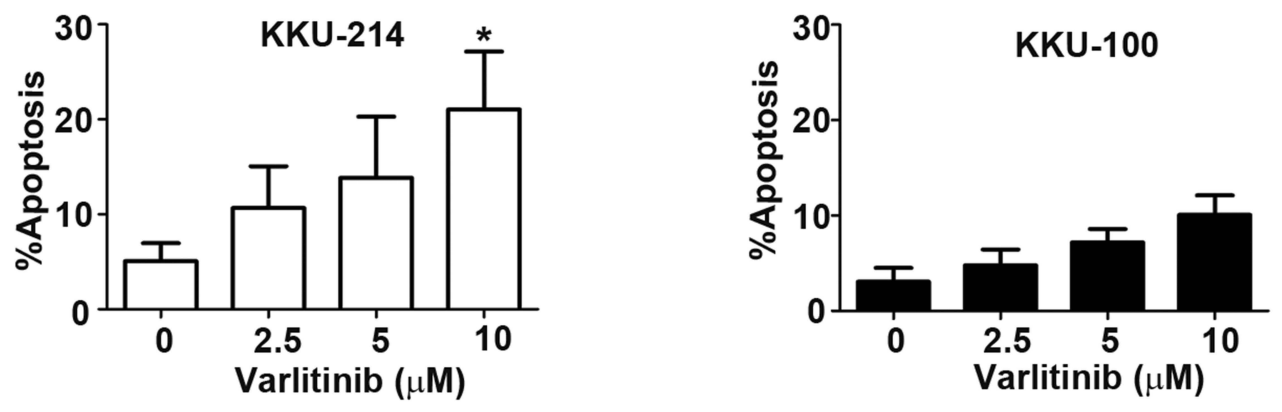

C

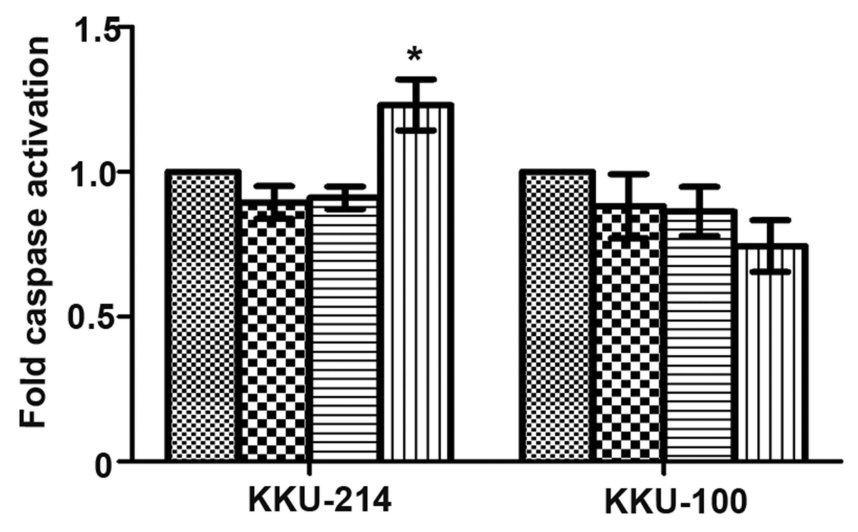

冈 Control

W Varlitinib $2.5 \mu \mathrm{M}$

国 Varlitinib $5 \mu \mathrm{M}$

血 Varlitinib $10 \mu \mathrm{M}$

Figure 4 Varlitinib induces CCA cell apoptosis.

Notes: (A and B) KKU-2I4 and KKU-100 cells were treated with varlitinib at the indicated concentrations after which the apoptotic cells were counted using the AnnexinV-FLUOS staining kit and flow cytometry. Live cells stained annexin V-/PI- (left bottom panel) and apoptotic cells stained annexin V+/PI- or annexin V+/PI+ (right bottom and right top panel). (C) A caspase-3 activity assay was performed on KKU-2I 4 and KKU-I00 cells after treatment with the designated concentrations for 72 hrs. Data in (B and $\mathbf{C}$ ) are represented as mean \pm SD of three independent experiments. Asterisks indicate statistical significance $(\mathrm{P}<0.00 \mathrm{I})$ compared to the control, analyzed by one-way ANOVA.

nude mice after which the mice were administrated with varlitinib at $25 \mathrm{mg} / \mathrm{kg}, 50 \mathrm{mg} / \mathrm{kg}$, or $100 \mathrm{mg} / \mathrm{kg}$ and vehicle (1:9 NMP: Polyetheneglycon) for the control group once daily by oral gavage for 15 days. The results showed that $50 \mathrm{mg} / \mathrm{kg}$ and $100 \mathrm{mg} / \mathrm{kg}$ of varlitinib significantly suppressed tumor growth, while $25 \mathrm{mg} / \mathrm{kg}$ had no significant effects (Figure 7A and B and Figure S1). Varlitinib-associated toxicity was not observed during the entire treatment period. Histological features of the internal organs, including lung, liver, kidney and spleen, indicated no signs of toxicity (Figure $\mathrm{S} 2, \underline{\mathrm{S} 3}, \underline{\mathrm{S} 4}$ and $\underline{\mathrm{S} 5}$ ), and biochemical analyses of liver and kidney function revealed 
A
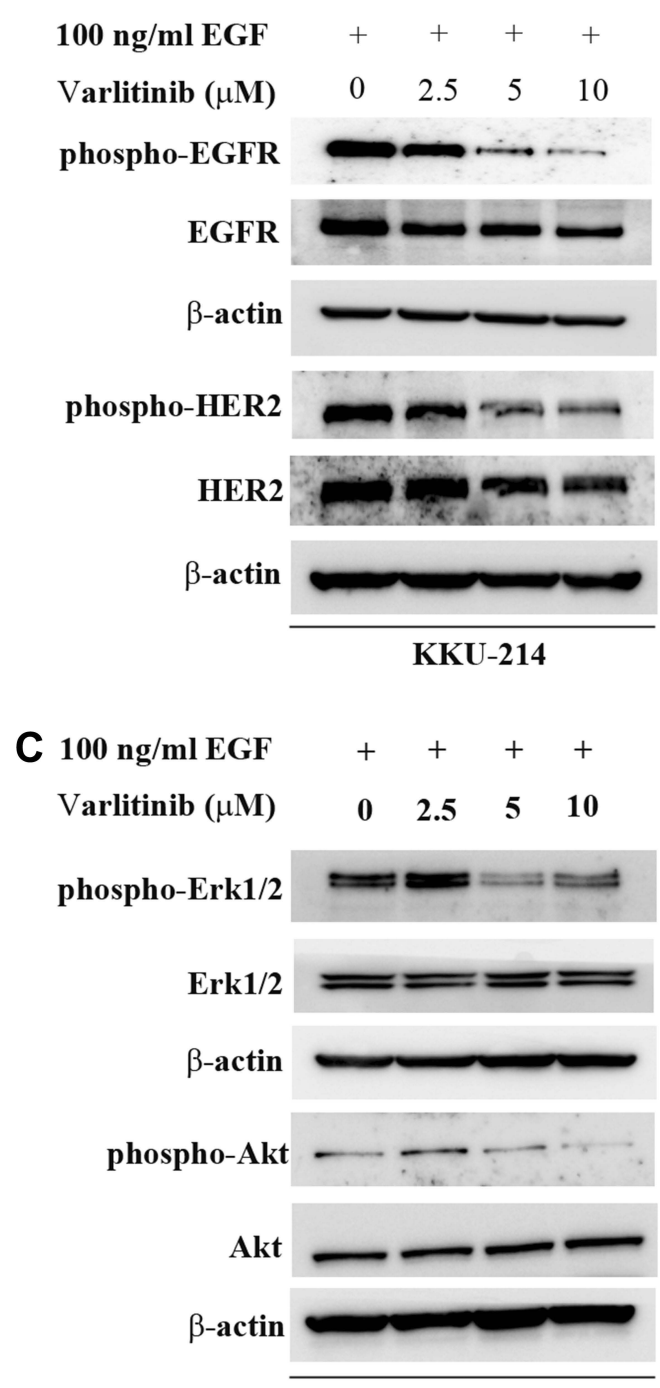

KKU-214
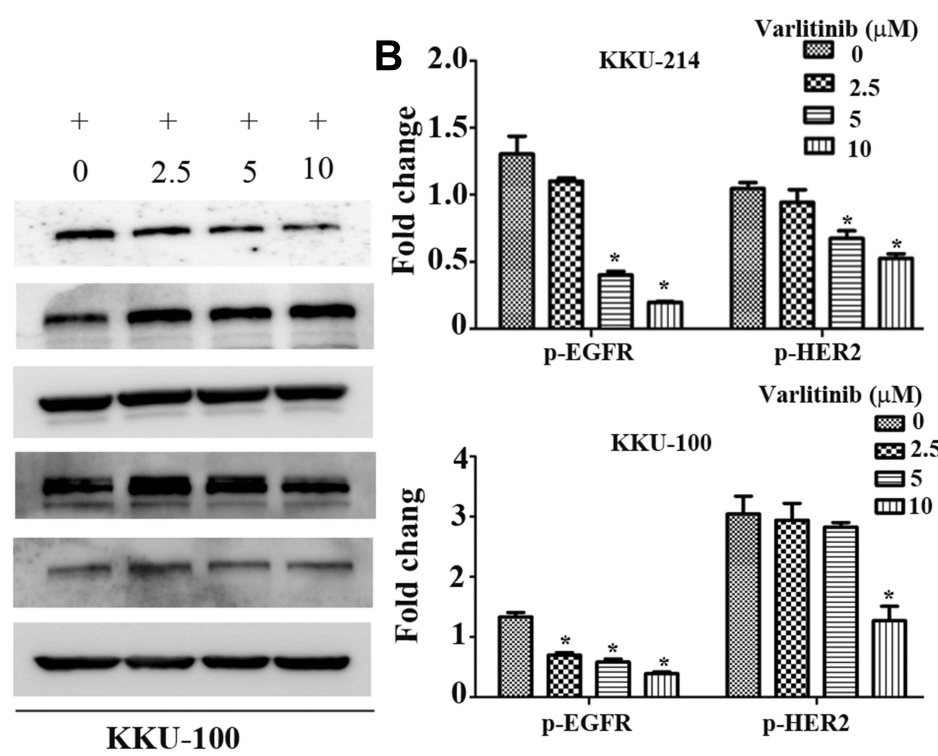

p-EGFR
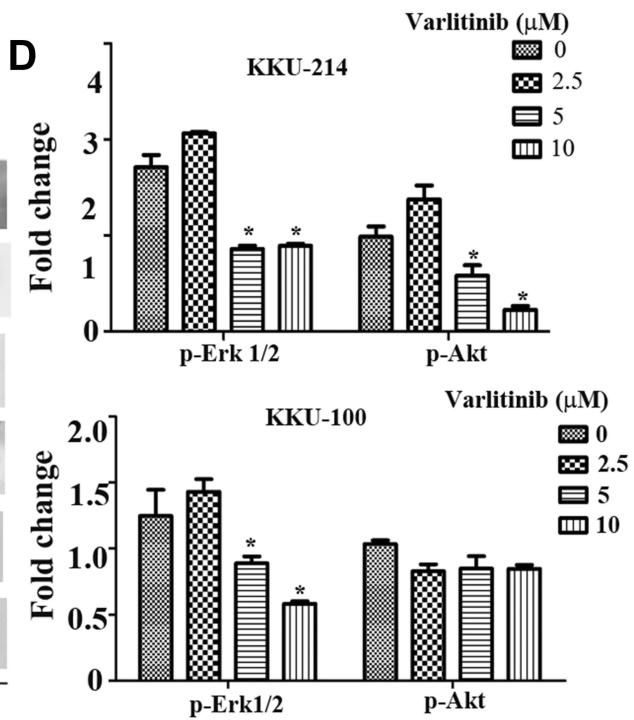

Figure 5 Molecular mechanisms by which varlitinib suppresses CCA cell growth. KKU-2I4 and KKU-I00 cells were exposed to varlitinib at the indicated concentrations for $24 \mathrm{hrs}$, then induced with $100 \mathrm{ng} / \mathrm{mL}$ of EGF for 30 mins, proteins expression level were determined by Western blot analysis.

Notes: (A) The activation of EGFR and HER2. (B) The expression of p-EGFR and p-HER2 after normalized with total EGFR, HER2 and $\beta$-actin. (C) The activation the downstream signaling molecules Erkl/2 and Akt. (D) The expression of p-Erkl/2 and p-Akt after normalized with total Erkl/2, AKT and $\beta$-actin. Data in (B and D) are represented as mean $\pm S D$ of three independent experiments. Asterisks indicate statistical significance $(p<0.00 \mathrm{I})$ compared to the control, analyzed by one-way ANOVA.

no differences between the control and the varlitinib treatment groups (Table S2). Nevertheless, the bodyweight of some mice in the control group was significantly reduced when tumor size reached $>15 \mathrm{~mm}$ in diameter when compared to the treatment groups (Figure 7C).

Immunostaining of the Ki67 proliferation marker was performed to confirm the anti-proliferation activity of varlitinib. The number of Ki67 nuclei stained tumor cells in varlitinib treated mice $(50 \mathrm{mg} / \mathrm{kg}$ and $100 \mathrm{mg} / \mathrm{kg}$ ) was significantly decreased when compared to the control group (Figure 7D). Moreover, apoptosis induction activity of varlitinib was detected by immunohistochemistry using TUNEL. Cell apoptosis was significantly higher in varlitinib $(50 \mathrm{mg} / \mathrm{kg}$ and 100 $\mathrm{mg} / \mathrm{kg}$ ) treated tumors than in the control group (Figure 7E).

\section{Tumor Metabolism in the Varlitinib- Treated CCA Xenograft Model}

Since varlitinib demonstrated growth inhibitory effect both in vitro and in animal model. Therefore, we further explore varlitinib response marker that correlated with CCA growth reduction after varlitinib treatment. The NMR-based metabolic profiling was carried out for CCA 
A

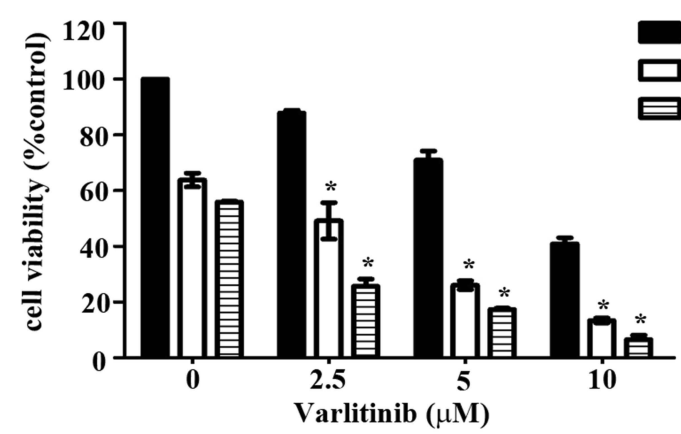

B

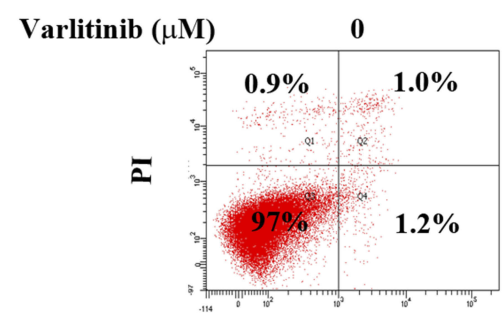

Varlitinib $(\mu \mathbf{M})$

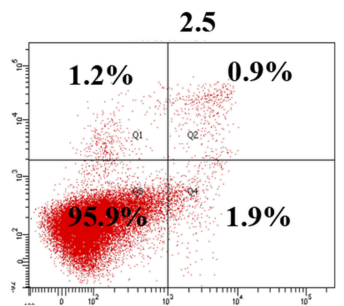

BKM-120 0 nM

BKM-120 1 nM

BKM-120 2 nM

BM-120 2 nM

0
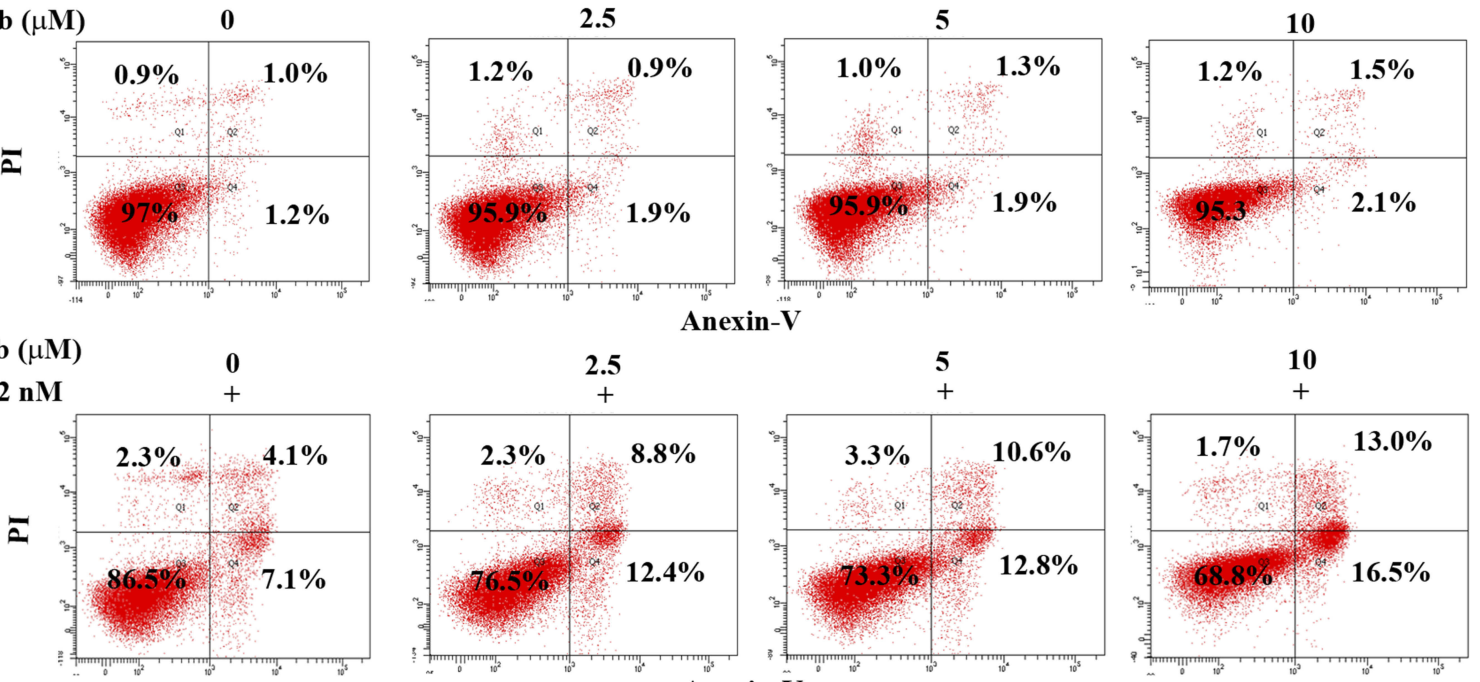

Anexin-V

C

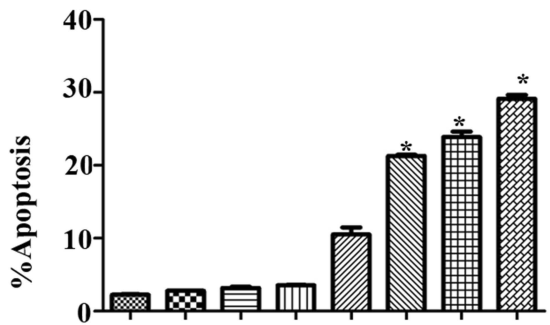

$\begin{array}{lllllllll}\text { Varlitinib }(\mu \mathrm{M}) & 0 & 2.5 & 5 & 10 & 0 & 2.5 & 5 & 10\end{array}$

$\begin{array}{llllllllll}\text { BKM-120 (nM) } & 0 & 0 & 0 & 0 & 2 & 2 & 2 & 2\end{array}$

E

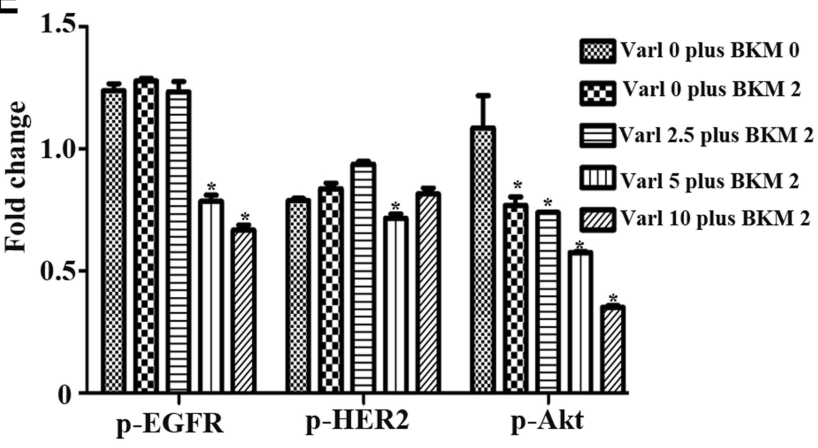

D
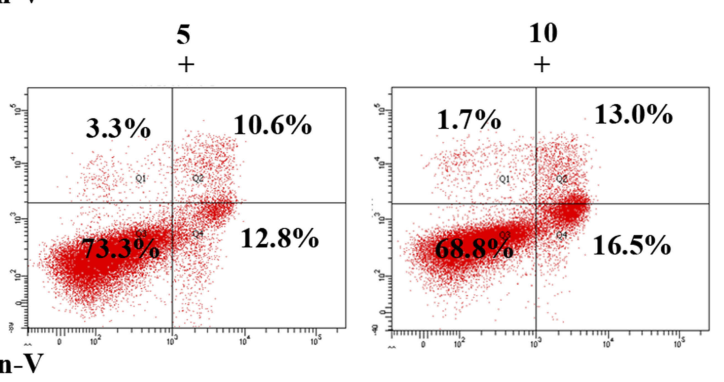

Anexin-V

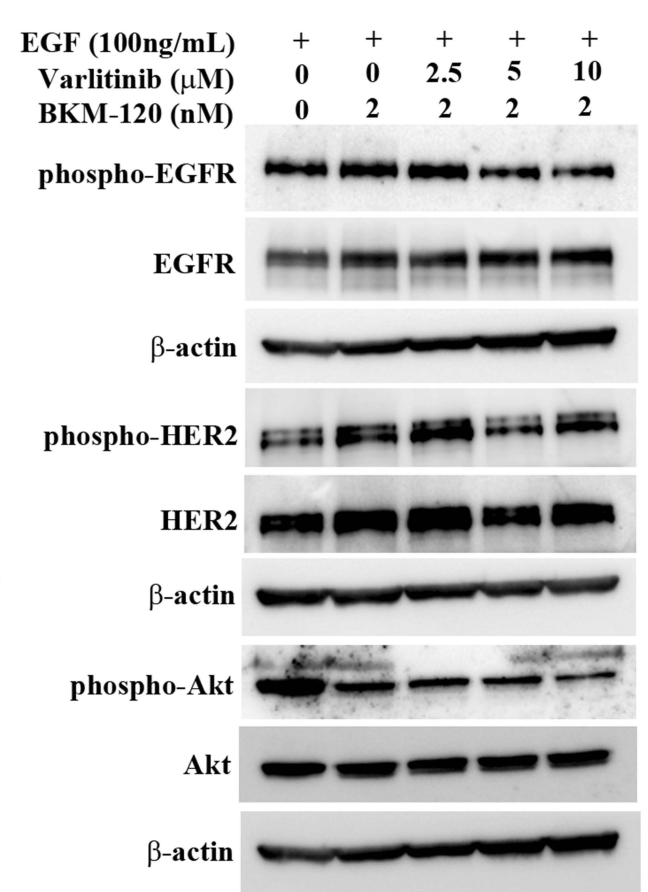

Figure 6 A combination of varlitinib with BKM-I20 potentiates the cytotoxicity of varlitinib on KKU- 100 cells.

Notes: (A) KKU-I00 cells were treated with varlitinib, BKM-120, or varlitinib combined with BKM- 120 at the indicated concentrations for 72 hrs after which cell proliferation was measured by SRB assay. (B and C) KKU-100 cells were exposed to varlitinib alone or varlitinib combined with BKM-120 at the indicated concentrations for 72 hrs after which cell apoptosis was assessed using the Annexin-V-FLUOS staining kit and flow cytometry. (D) Molecular analysis of varlitinib combined with BKM-I20 in KKU-I00 cells was identified by Western blot. (E) The expression level of p-EGFR, p-HER2, p-Akt after normalized with total form and $\beta$-actin. Data in $(\mathbf{A}, \mathbf{C}$ and $\mathbf{E})$ are represented as mean \pm SD of three independent experiments. Asterisks indicate statistical significance $(P<0.001)$ compared to the control, analyzed by two-way ANOVA and one-way ANOVA, respectively. 
A

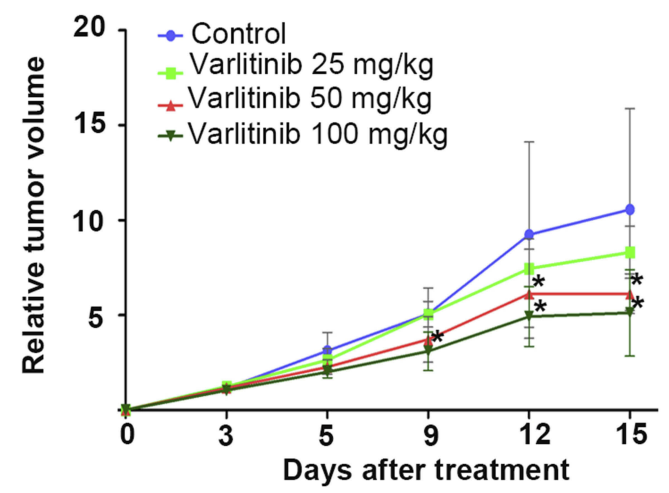

C

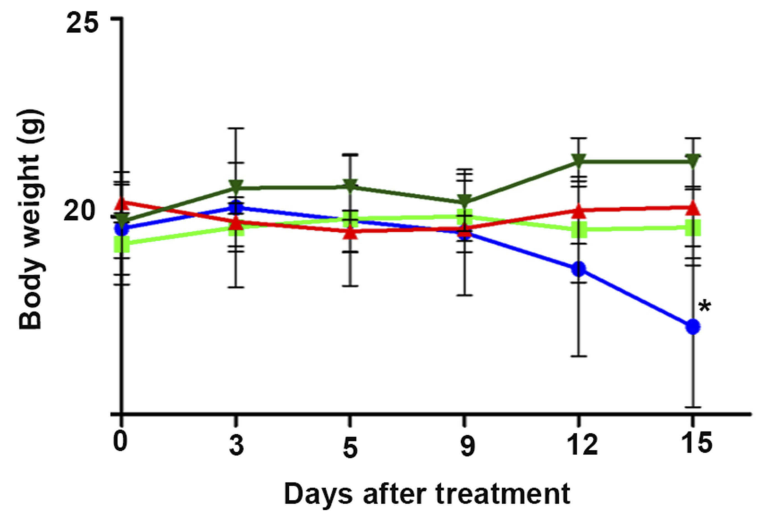

B

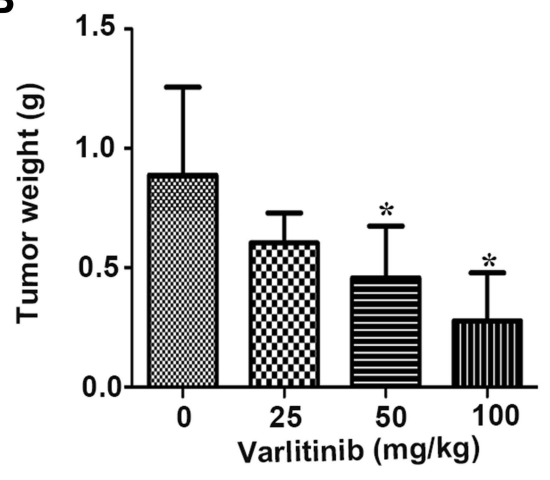

- Contol

- Varlitinib $25 \mathrm{mg} / \mathrm{kg}$

$\leftarrow$ Varlitinib $50 \mathrm{mg} / \mathrm{kg}$

$\rightarrow$ Valitinib $100 \mathrm{mg} / \mathrm{kg}$
D

Control

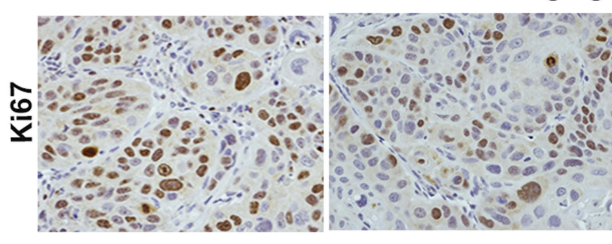

E

Control

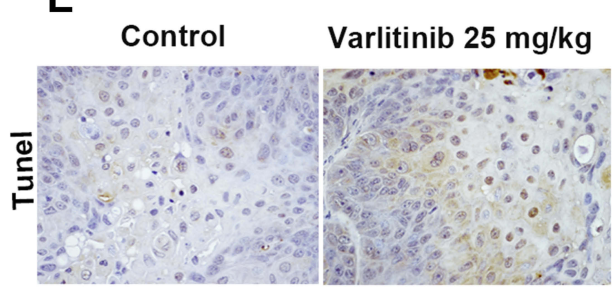

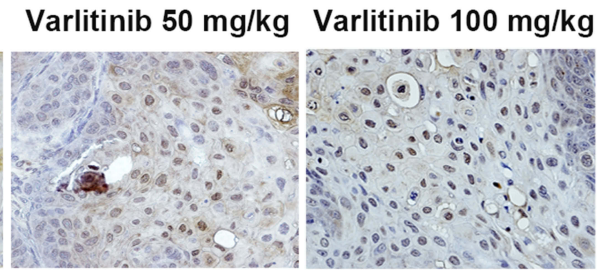
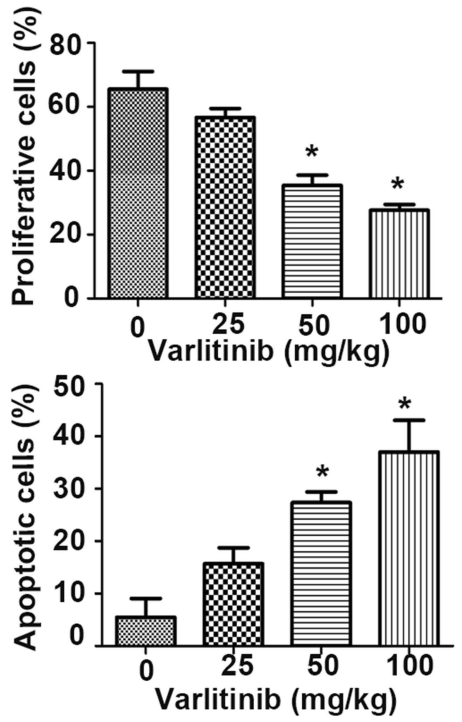

Figure 7 Anti-tumor activity of varlitinib on CCA inoculated mice.

Notes: KKU-2I 4 was implanted in mice which were orally administrated the vehicle, $25-50 \mathrm{mg} / \mathrm{kg}$ or $100 \mathrm{mg} / \mathrm{kg}$ of varlitinib daily ( $\mathrm{n}=6 / \mathrm{group})$. (A) Tumor growth and (B) tumor weight of mice that received the vehicle, $25 \mathrm{mg} / \mathrm{kg}, 50 \mathrm{mg} / \mathrm{kg}$ or $100 \mathrm{mg} / \mathrm{kg}$ of varlitinib for 15 days. (C) The average body weight of mice in each group. (D) Proliferative cells in nude mouse tumor tissue sections were determined by immunostaining of Ki67, magnification X400. (E) Apoptotic cells were detected by TUNEL assay, magnification X400. Data in (A) and (C) are represented as mean \pm SD and analyzed by two-way ANOVA, data in (B, D and E) are represented as mean \pm SD and analyzed by independent sample $t$-test. Asterisks indicate statistical significance $(P<0.00 \mathrm{I})$ compared to the control.

inoculated mouse tissue extracts in order to identify the metabolites that correlate with varlitinib responses and metabolic pathways that were associated with CCA growth inhibition effect of varlitinib in animal model.
The representative NMR spectra of control, and varlitinib $(25 \mathrm{mg} / \mathrm{kg}, 50 \mathrm{mg} / \mathrm{kg}$ and $100 \mathrm{mg} / \mathrm{kg})$-treated CCA inoculated mouse tumor tissues revealed numerous signals from metabolites as shown in Figure 8A. 

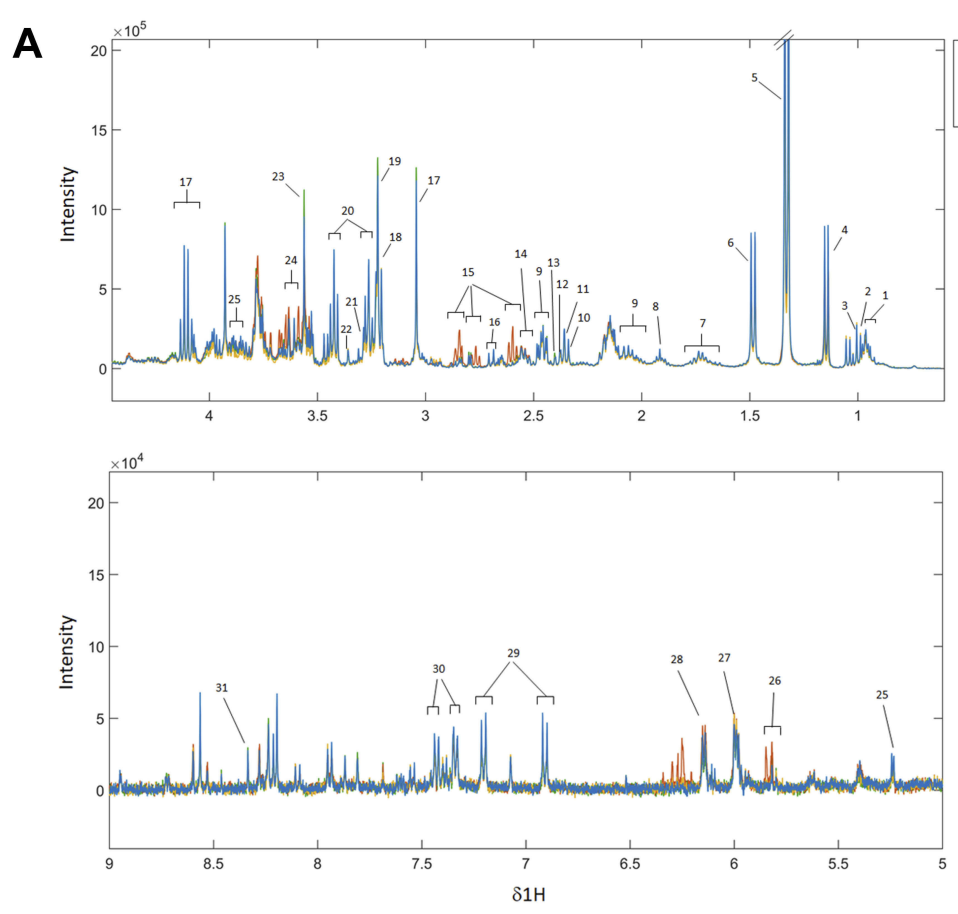

D

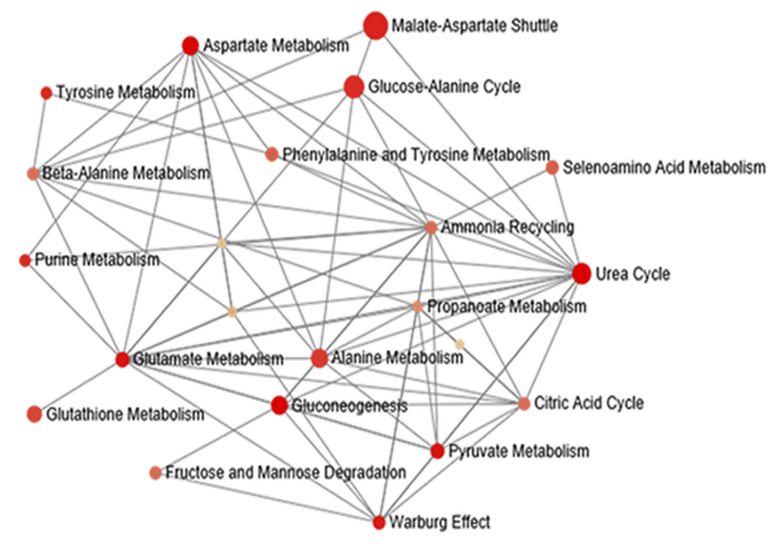

E

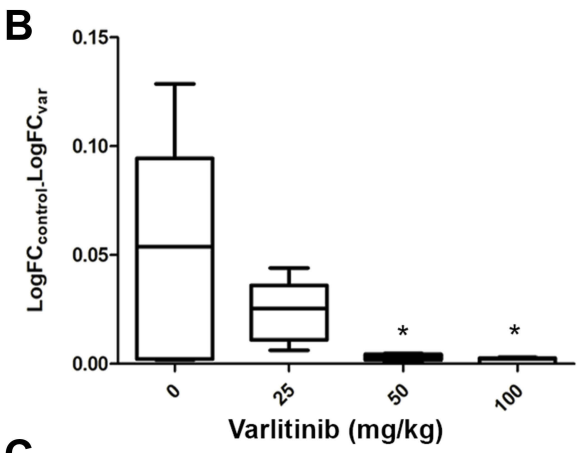

C

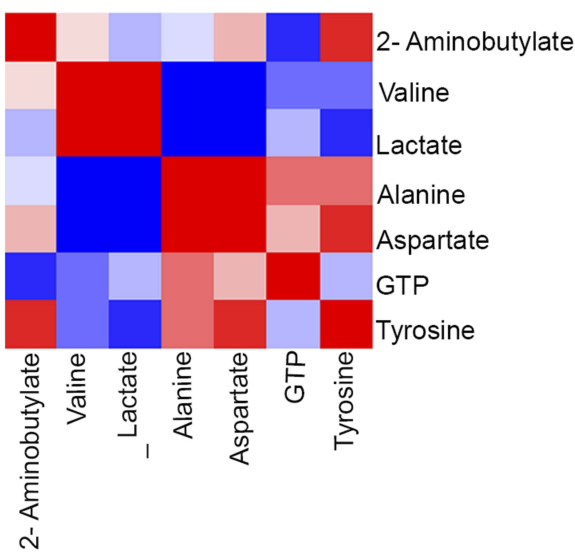

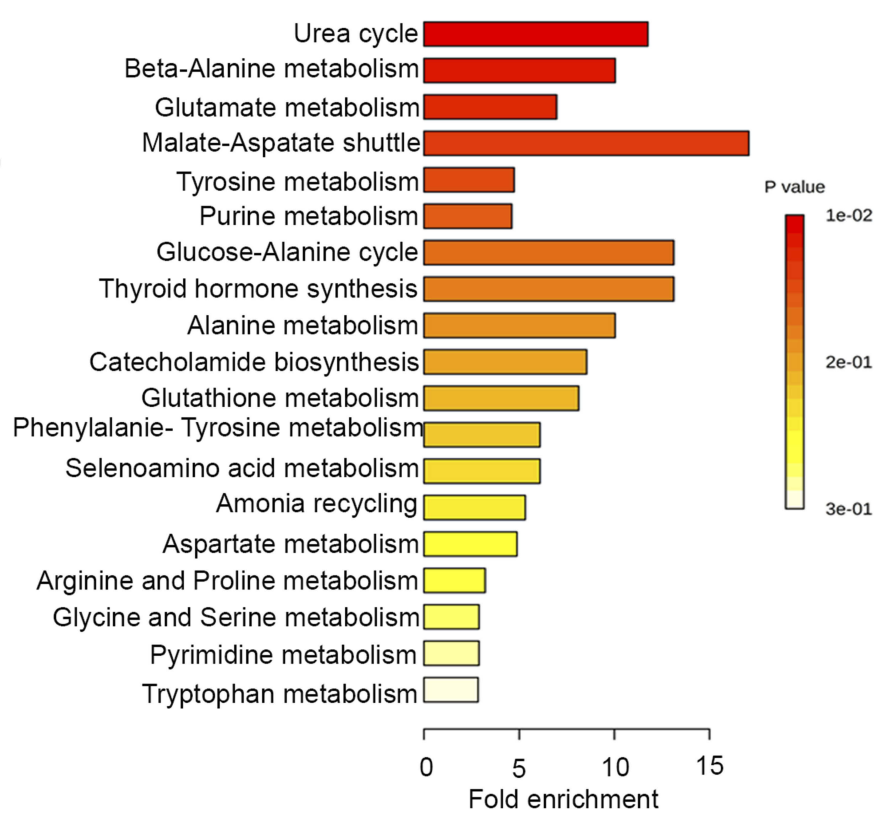

Figure 8 Tumor metabolism of varlitinib-treated CCA xenograft model.

Notes: (A) Median spectrum of IH NMR spectrum of nude mice's tissue (Key metabolites: (I) Leucine, (2) 2-aminobutyrate, (3) Valine, (4) Propylene glycol, (5) Lactate, (6) Alanine, (7) Arginine, (8) Acetate, (9) Glutamine, (10) Glutamate, (II) Pyruvate, (12) Oxaloacetate, (13) Succinate, (14) Citrate, (15) Aspartate, (16) Methionine, (17) Creatine, (18) Choline, (19) Phosphorylcholine, (20) Taurine, (2I) TMAO, (22) Methanol, (23) Glycine, (24) Glycerol, (25) Glucose, (26) Uracil, (27) GTP, (28) ATP, (29) Tyrosine, (30) Phenylalanine, (31) Inosine. (B) Box plots of aspartate expressed as a fold change of more than I.2 between normal and cancerous tissue. Asterisks indicate statistical significance $(\mathrm{p}<0.00 \mathrm{I})$ analyzed by Student's $t$-test. $(\mathbf{C})$ Heatmap analysis represents the metabolic phenotype change after varlitinib treatment; the heatmaps were constructed based on the correlation between the intensity peaks of assigned metabolites. Color key indicates metabolite expression value, blue represents a positive correlation, red a negative correlation. (D) Pathway-network analysis showed the relationship between the reflected metabolic pathways; the color and size of each pathway represents the pathway's impact and fold enrichment, respectively. (E) The significant metabolites set enrichment, the most impacted pathways that have statistically significant scores are indicated with red and the lengths of each bar are calculated based on the pathway value using Metaboanalyst 3.0. 
Then, peak intensities of the detectable metabolites were calculated for the fold change and statistically tested in each varlitinib-treated group compared the control counterpart. The results are reported in Table S3. We found seven candidate metabolites whose levels were significantly changed for each concentration of varlitinib (eg, 2-aminobutylate, valine, lactate, alanine, aspartate, GTP and tyrosine). Interestingly, only aspartate was significantly decreased $(\mathrm{P}<0.05)$ with a fold change of more than 1.2 at $50 \mathrm{mg} / \mathrm{kg}$ and $100 \mathrm{mg} / \mathrm{kg}$ varlitinib (Figure 8B).

To explore the metabolic pathways associated with the metabolic alteration after varlitinib treatment, heat map analysis and pathway analysis were performed. We found the correlation between the metabolic candidates changed, demonstrating the biochemical phenotypic changes after varlitinib treatment (Figure 8C). Pathway-network analysis revealed several relevant metabolic pathways that the candidate metabolites were implicated of which the urea cycle is the most closely associated followed by beta-alanine metabolism and glutamate metabolism (Figure 8D). However, when using pathway analysis of fold enrichment, we found that malate-aspartate shuttle showed the highest fold enrichment, indicating that this shuttle is the metabolic pathway that exhibited most drastic change at the metabolic level after varlitinib treatment (Figure 8E).

\section{Discussion}

Epidermal growth factor receptors (EGFR), also called the HER or ErbB receptor family, is a family of receptor tyrosine kinases that is composed of four members: HER1 (EGFR), HER2 (c-erbB-2), HER3 (c-erbB-3) and HER4 (c-erbB4). ${ }^{7}$ These receptors activate multiple downstream pathways in response to extracellular ligands that, in turn, regulate diverse processes including cell proliferation, survival, differentiation and migration. ${ }^{8}$ Abnormal regulation and activation of these receptors are involved in the development and progression of many cancer types. ${ }^{9}$ A large number of studies have reported either the overexpression or amplification of EGFR and HER 2 in various malignancies, ${ }^{19,20}$ and there are several approved therapeutics targeting these mechanisms (eg, Herceptin, Iressa). ${ }^{21,22}$ Moreover, overexpression of HER3 is involved in primary cancers and in cultured cells, including breast, ovarian, prostate, colon, pancreas, stomach, oral cavity and lung cancer, as well as EGFR inhibitor resistance. HER4 is associated with breast cancer and can be used in the prediction of the tamoxifen response. ${ }^{23,24}$ In CCA, aberrant expression and/or activation of the HER receptor family has been reported. ${ }^{10,25-27}$ Our previous study revealed the activation of the HER receptor family in CCA using phospho-kinase array analysis. In the HER receptor family, EGFR was found to be activated in both human CCA tissues and CCA cell lines. ${ }^{12}$ A recent study of CCA demonstrated the overexpression of EGFR, which was associated with a poor prognosis of CCA patients. ${ }^{13}$ Targeting EGFR using nimotuzumab inhibits CCA cell metastasis through the suppression of the epithelial-mesenchymal-transition mechanism. Furthermore, HER2 gene amplification has been noted in liver fluke associated CCA. ${ }^{14}$ Thus, these receptor kinases are promising targets for anti-cancer drug development in CCA.

Our study provides a pre-clinical evaluation of varlitinib for first line CCA treatment. First, we identified the expression of the HER receptor family in four CCA cell lines (KKU-214, KKU-213, KKU-156, KKU-100) and in non-malignant cholangiocyte, MMNK-1 cell. The results showed that KKU-214 had the highest expression of EGFR, HER2 and HER3, KKU-100 had a high expression of EGFR and HER2; HER4 was also found very low level in all tested CCA cell lines. On the other hand, low expression level of HER family was detected in MMNK1 cell. A cytotoxicity test revealed that $\mathrm{IC}_{50}$ values of KKU-214, KKU-213 and KKU-156 cells were within a similar range $(4 \mu \mathrm{M})$, while KKU-100 showed a higher $\mathrm{IC}_{50}$ value than the others $(8 \mu \mathrm{M})$. Interestingly, varlitinib showed low toxicity on non-malignant cholangiocyte which is indicated by higher $\mathrm{IC}_{50}$ value of varlitinib on MMNK-1 cell over CCA cell lines. Varlitinib is highly $(>99 \%)$ but reversibly bound to plasma proteins in vitro; hence, the $\mathrm{IC}_{50}$ of varlitinib in serum-enriched conditions is expected to be much higher.

Based on EGFR and HER2 protein expression levels and $\mathrm{IC}_{50}$ values, varlitinib response should be correlated the expression of HER family especially EGFR and HER2. However, KKU-214 and KKU-100 cells which had high expression of EGFR and HER2 showed the different response patterns to varlitinib. Therefore, these two CCA cell lines were selected for the next set of experiments.

Varlitinib was shown to significantly inhibit KKU-214 cell proliferation and enhance cell death in a dose-dependent manner. However, only the $10 \mu \mathrm{M}$ concentration of varlitinib could significantly suppress KKU-100 growth and it had no apoptotic induction effect on this cell line. Molecular analysis using Western blot demonstrated that varlitinib can inhibit the activation of EGFR, HER2, Erk1/2 and Akt upon stimulation with EGF, whereas in KKU-100 cells varlitinib can 
inhibit the activation of EGFR and Erk1/2, although the activation of HER2 and Akt was still observed. This may be caused by a compensatory mechanism in the KKU-100 cells. A possible explanation will be discussed in the following section.

HER2 can form a heterodimer not only with the HER receptor family but also with other RTKs such as IGF-1R. In breast cancer, the IGF-1R/HER2 complex can potentiate $\mathrm{PI} 3 \mathrm{~K} / \mathrm{Akt}$ signaling that leads to trastuzumab resistance. ${ }^{28}$ Moreover, the recent study revealed that the acquired lapatinib resistance mechanism in HER2 positive gastric cancer cells caused by the interplay between HER2 and MET receptor resulted from downregulation of FOXO1. Therefore, constitutive activation of HER2 after exposure to varlitinib in KKU-100 cells may occur due to activation of a compensatory signaling pathway by forming a heterodimer between HER2 with other RTKs. However, this compensatory mechanism in CCA is still unclear and should be further investigated. ${ }^{29}$ The other compensatory mechanism involves downstream alterations and mutations. Inherited resistance due to KRAS mutations may be a reason for the poor response to varlitinib in KKU-100 cells. KRAS mutations, especially the G12D mutation, are strong predictors of a lack of response to EGFR inhibition. Mutation of KRAS can cause persistent activation of ras as well as its downstream effector molecules, such as Akt, even resulting in a lack of response to the EGFR inhibitor in patients with non-small cell lung cancer. ${ }^{30}$ Likewise, KKU-100, but not the other three CCA cell lines, possesses a G12D mutation in exon $2^{31}$ that might lead to the poor susceptibility of this cell to varlitinib. A combination of EGFR inhibitor with a downstream effector molecule inhibitor such as PI3K inhibitor or MEK inhibitor, can overcome this phenomenon. ${ }^{32}$ In this study, a combination of varlitinib with PI3K inhibitor which has previously been reported to enhance CCA cell apoptosis both in cell lines and an animal model, ${ }^{33}$ led to an improved response of KKU-100 cells to varlitinib. Previous studies ${ }^{33}$ have shown that KKU-100 is a CCA cell line that is exquisitely sensitive to BKM-120 inhibition with a $\sim 10$-fold lower $\mathrm{IC}_{50}$ compared to other CCA cell lines such as M214. The results showed that varlitinib combined with BKM-120 can significantly inhibit KKU-100 cell growth and induce cell death when compared to treatment with varlitinib or BKM-120 alone. Moreover, molecular analysis showed that the activation of Akt was decreased when KKU-100 cells were treated with varlitinib combined with BKM-120. This indicates that inhibition of EGFR/HER2 signaling by targeting Akt can overcome the poor susceptibility of this cell subtype of CCA with KRAS G12D mutations and can potentially serve as a treatment regimen for patients who do not respond to EGFR inhibitor treatment.

We further evaluated the anti-tumor activity of varlitinib in KKU-214 implanted nude mice. Our findings showed that varlitinib at $50 \mathrm{mg} / \mathrm{kg}$ and $100 \mathrm{mg} / \mathrm{kg}$ can significantly suppress tumor growth in mice when compared to control mice, without noticeable toxicity. The bodyweight of the control mice was significantly decreased at day 15 of treatment when tumor size reached more than $1.5 \mathrm{~cm}$ diameter. The bodyweight of varlitinibtreated mice was similar throughout the duration of treatment.

In order to determine the safety of varlitinib in animals, mouse serum was used to determine the liver and kidney relevant parameters AST, ALT and creatinine. The serum AST, ALT and creatinine levels of mice from each group were similar (Table S2). Moreover, the histological features of the mouse organs, including lung, liver, kidney and spleen, were checked by a pathologist. They also showed minimal or no toxic effect in normal cells (supplemental data), indicating no adverse effect of varlitinib on animals.

The growth inhibitory effect of varlitinib is indicated by immunostaining of the proliferation marker Ki67. Our result showed that the percentage of Ki67 positive nuclear staining cells in tumor tissues of the $50 \mathrm{mg} / \mathrm{kg}$ and 100 $\mathrm{mg} / \mathrm{kg}$ varlitinib-treated mice was significantly decreased when compared with the control group. Moreover, the percentage of apoptotic cells was significantly increased in tumor tissue from 50 to $100 \mathrm{mg} / \mathrm{kg}$ varlitinib-treated mice when compared with tissue from control animals. These results indicate the anti-tumor potential of varlitinib using the CCA animal model.

We also accessed tumor metabolism after varlitinib treatment in CCA xenograft tumor tissue extracted by using ${ }^{1} \mathrm{H}-$ NMR analysis. Our study revealed a number of metabolites (Figure 8A and Table S3) which are associated with varlitinib treatment response. Among the detectable metabolites, we found significant metabolic changes after varlitinib treatment in 2-aminobutylate, valine, lactate, alanine, aspartate, GTP and tyrosine. Interestingly, aspartate is the metabolite that changed significantly, with a fold change of more than 1.2 in the $50 \mathrm{mg} / \mathrm{kg}$ and $100 \mathrm{mg} / \mathrm{kg}$ groups (Figure $8 \mathrm{~B}$ and Table S3). This is consistent with an in vivo anti-cancer effect of varlitinib. Doses of $50 \mathrm{mg} / \mathrm{kg}$ and $100 \mathrm{mg} / \mathrm{kg}$ of varlitinib can significantly inhibit tumor growth in the CCA xenograft 
model (Figure 7A and B). Thus, aspartate is a potential metabolite correlating with varlitinib treatment response.

Aspartate is the ionic form of aspartic acid, a nonessential amino acid which is frequently synthesized through the transamination of oxalate in the human body. Aspartate acquisition has been reported as an endogenous metabolic limitation for cancer cell proliferation. Aspartate enables cell proliferation via mitochondrial respiration, which is an important mechanism providing an electron acceptor for aspartate synthesis. ${ }^{34,35}$ This suggests that aspartate plays role in tumor cell proliferation with decreasing levels which causes tumor growth reduction to occur. A metabolomic study in head and neck squamous cell carcinoma with EGFR inhibitor acquired resistant three-dimensional (3-D) spheroids and xenograft model tissue showed a significantly increased aspartate level when compared with the EGFR TKI sensitive model, suggesting that aspartate can serve as a potential metabolic biomarker for drug response.

We further identify the metabolic pathways that reflect the metabolic changes occurring after varlitinib treatment. We found that the varlitinib treatment influences the urea cycle followed by beta-alanine metabolism and glutamate metabolism. Interestingly, pathway analysis of fold enrichment showed that the malate-aspartate shuttle is the most influenced metabolic pathway after varlitinib treatment. This is consistent with the hypothesis that aspartate is the key metabolite biomarker correlating with varlitinib response. Aspartate plays a role in several metabolic pathways. It acts as an amino group donor in the urea formation process of the urea cycle, producing beta-alanine through the action of glutamate decarboxylase. ${ }^{36,37}$ Aspartate constitutes a pivotal component of the malateaspartate shuttle that enables energy production in cells, ${ }^{38}$ suggesting the importance of the metabolic pathway involving aspartate after varlitinib treatment.

Taken together, varlitinib inhibits CCA cell proliferation and induces apoptosis both in vitro and in vivo, indicating its potential as a therapeutic agent for CCA. Varlitinib was also found to be a safe regimen with minimal toxicity observed in the animals. A combination of varlitinib with the PI3K inhibitor BKM-120 potentiates the cytotoxicity of varlitinib in the less sensitive KKU-100 CCA cell line. Moreover, the metabolomic study suggests aspartate as the response biomarker for varlitinib treatment. Knowledge from this study provides non-clinical data in support of clinical trials of varlitinib in CCA. However, varlitinib was recently declared to fail in CCA second line in clinical trial. According to our preclinical study results, we would like to recommend the use of varlitnib in combination with $\mathrm{PI} 3 \mathrm{~K}$ inhibitor or cytotoxic drug such as gemcitabine in order to increase the efficacy of varlitinib in CCA treatment.

\section{Acknowledgment}

The authors thank Professor Trevor N. Petney for editing the initial submission via Publication Clinic KKU, Thailand.

\section{Disclosure}

This work was supported by the Graduate School, Khon Kaen University to Hasaya Dokduang and ASLAN Pharmaceuticals, Singapore to Watcharin Loilome. The authors report no other conflicts of interest in this work.

\section{References}

1. Elkins DB, Haswell-Elkins MR, Mairiang E, et al. A high frequency of hepatobiliary disease and suspected cholangiocarcinoma associated with heavy Opisthorchis viverrini infection in a small community in north-east Thailand. Trans R Soc Trop Med Hyg. 1990;84 (5):715-719. doi:10.1016/0035-9203(90)90159-C

2. Sripa B, Pairojkul C. Cholangiocarcinoma: lessons from Thailand. Curr Opin Gastroenterol. 2008;24(3):349-356.

3. Khan SA, Taylor-Robinson SD, Toledano MB, Beck A, Elliott P, Thomas HC. Changing international trends in mortality rates for liver, biliary and pancreatic tumours. J Hepatol. 2002;37(6):806-813.

4. Khuntikeo N, Pugkhem A, Titapun A, Bhudhisawasdi V. Surgical management of perihilar cholangiocarcinoma: a Khon Kaen experience. J Hepatobiliary Pancreat Sci. 2014;21(8):521-524.

5. Titapun A, Pugkhem A, Luvira V, et al. Outcome of curative resection for perihilar cholangiocarcinoma in Northeast Thailand. World $J$ Gastrointest Oncol. 2015;7(12):503-512.

6. de Groen PC, Gores GJ, LaRusso NF, Gunderson LL, Nagorney DM. Biliary tract cancers. N Engl J Med. 1999;341(18):1368-1378.

7. Bazley LA, Gullick WJ. The epidermal growth factor receptor family. Endocr Relat Cancer. 2005;12(Suppl 1):S17-27.

8. Wieduwilt MJ, Moasser MM. The epidermal growth factor receptor family: biology driving targeted therapeutics. Cell Mol Life Sci. 2008;65(10):1566-1584.

9. Roskoski R Jr. The ErbB/HER family of protein-tyrosine kinases and cancer. Pharm Res. 2014;79:34-74.

10. Sirica AE. Role of ErbB family receptor tyrosine kinases in intrahepatic cholangiocarcinoma. World j Gastroenterol. 2008;14(46):70337058.

11. Yang X, Wang W, Wang C, et al. Characterization of EGFR family gene aberrations in cholangiocarcinoma. Oncol Rep. 2014;32(2):700708.

12. Dokduang H, Juntana S, Techasen A, et al. Survey of activated kinase proteins reveals potential targets for cholangiocarcinoma treatment. Tumour Biol. 2013;34(6):3519-3528.

13. Padthaisong S, Thanee M, Techasen A, et al. Nimotuzumab inhibits cholangiocarcinoma cell metastasis via suppression of the epithelialmesenchymal transition process. Anticancer Res. 2017;37(7):35913597.

14. Jusakul A, Cutcutache I, Yong CH, et al. Whole-Genome and epigenomic landscapes of etiologically distinct subtypes of cholangiocarcinoma. Cancer Discov. 2017;7(10):1116-1135. 
15. Coelho SC, Reis DP, Pereira MC, Coelho MAN. Gold nanoparticles for targeting varlitinib to human pancreatic cancer cells. Pharmaceutics. 2018;10(3). doi:10.3390/pharmaceutics10030091

16. Liu CY, Chu PY, Huang CT, et al. Varlitinib downregulates HER/ ERK signaling and induces apoptosis in triple negative breast cancer cells. Cancers. 2019;11(1). doi:10.3390/cancers11010105

17. Miknis G, Wallace E, Lyssikatos J, et al. ARRY-334543, A potent, orally active small molecule inhibitor of EGFR and ErbB-2. Cancer Res. 2005;65:801.

18. Varlitinib. Varlitinib Pipeline. ASLAN Pharm. 2017. http://aslan pharma.com/drug/aslan001/.

19. Hirsch FR, Varella-Garcia M, Cappuzzo F. Predictive value of EGFR and HER2 overexpression in advanced non-small-cell lung cancer. Oncogene. 2009;28(Suppl 1):S32-37.

20. Kavarthapu R, Dufau ML. Role of EGF/ERBB1 in the transcriptional regulation of the prolactin receptor independent of estrogen and prolactin in breast cancer cells. Oncotarget. 2016;7(40):65602-65613.

21. Awaya H, Takeshima Y, Furonaka O, Kohno N, Inai K. Gene amplification and protein expression of EGFR and HER2 by chromogenic in situ hybridisation and immunohistochemistry in atypical adenomatous hyperplasia and adenocarcinoma of the lung. J Clin Pathol. 2005;58(10):1076-1080.

22. Rimawi MF, Schiff R, Osborne CK. Targeting HER2 for the treatment of breast cancer. Annu Rev Med. 2015;66:111-128.

23. Jaiswal BS, Kljavin NM, Stawiski EW, et al. Oncogenic ERBB3 mutations in human cancers. Cancer Cell. 2013;23(5):603-617.

24. Mujoo K, Choi BK, Huang Z, Zhang N, An Z. Regulation of ERBB3/ HER3 signaling in cancer. Oncotarget. 2014;5(21):10222-10236.

25. Harder J, Waiz O, Otto F, et al. EGFR and HER2 expression in advanced biliary tract cancer. World $j$ Gastroenterol. 2009;15 (36):4511-4517.

26. Kawamoto T, Krishnamurthy S, Tarco E, et al. HER receptor family: novel candidate for targeted therapy for gallbladder and extrahepatic bile duct cancer. Gastrointest Cancer Res. 2007;1(6):221-227.
27. Yarden Y. The EGFR family and its ligands in human cancer. signalling mechanisms and therapeutic opportunities. Eur J Cancer. 2001;37(Suppl 4):S3-8

28. Lu Y, Zi X, Zhao Y, Mascarenhas D, Pollak M. Insulin-like growth factor-I receptor signaling and resistance to trastuzumab (Herceptin). J Natl Cancer Inst. 2001;93(24):1852-1857.

29. Park J, Choi Y, Ko YS, et al. FOXO1 suppression is a determinant of acquired lapatinib-resistance in HER2-positive gastric cancer cells through MET upregulation. Cancer Res Treat. 2018;50(1):239-254.

30. Engelman JA, Janne PA. Mechanisms of acquired resistance to epidermal growth factor receptor tyrosine kinase inhibitors in non-small cell lung cancer. Clin Cancer Res. 2008;14(10):2895-2899.

31. Saensa-Ard S, Leuangwattanawanit S, Senggunprai L, et al. Establishment of cholangiocarcinoma cell lines from patients in the endemic area of liver fluke infection in Thailand. Tumour Biol. 2017;39(11):1010428317725925.

32. Engelman JA, Chen L, Tan X, et al. Effective use of PI3K and MEK inhibitors to treat mutant Kras G12D and PIK3CA H1047R murine lung cancers. Nat Med. 2008;14(12):1351-1356.

33. Padthaisong $\mathrm{S}$, Dokduang $\mathrm{H}$, Yothaisong $\mathrm{S}$, et al. Inhibitory effect of NVP-BKM120 on cholangiocarcinoma cell growth. Oncology letters. 2018;16(2):1627-1633.

34. Sullivan LB, Gui DY, Hosios AM, Bush LN, Freinkman E, Vander Heiden MG. Supporting aspartate biosynthesis is an essential function of respiration in proliferating cells. Cell. 2015;162(3):552-563.

35. Sullivan LB, Luengo A, Danai LV, et al. Aspartate is an endogenous metabolic limitation for tumour growth. Nat Cell Biol. 2018;20 (7):782-788.

36. Lehninger ALNDL, Cox MM. Principles of Biochemistry. New York: W. H. Freeman; 2000.

37. MS D. DL-Aspartic acid. Organic Syntheses. 1950;4:55.

38. Wallace DC. Mitochondria and cancer. Nat Rev Cancer. 2012;12 (10):685-698.

\section{Publish your work in this journal}

Drug Design, Development and Therapy is an international, peerreviewed open-access journal that spans the spectrum of drug design and development through to clinical applications. Clinical outcomes, patient safety, and programs for the development and effective, safe, and sustained use of medicines are a feature of the journal, which has also been accepted for indexing on PubMed Central. The manuscript management system is completely online and includes a very quick and fair peer-review system, which is all easy to use. Visit http://www. dovepress.com/testimonials.php to read real quotes from published authors. 